1 Impact of commuting exposure to traffic-related air pollution on cognitive development in children walking to school

Mar Alvarez-Pedrerol1,2,3, loar Rivas ${ }^{1,2,3}$, Mònica López-Vicente ${ }^{1,2,3}$, Elisabet Suades-González 1,2,3,5,

4 David Donaire-Gonzalez ${ }^{1,2,3}$, Marta Cirach ${ }^{1,2,3}$, Montserrat de Castro ${ }^{1,2,3}$, Mikel Esnaola ${ }^{1,2,3}$, Xavier

5 Basagaña ${ }^{1,2,3}$, Payam Dadvand ${ }^{1,2,3}$, Mark Nieuwenhuijsen ${ }^{1,2,3}$, Jordi Sunyer ${ }^{1,2,3,4}$.

$6{ }^{1}$ ISGlobal, Centre for Research in Environmental Epidemiology (CREAL), Barcelona, Catalonia, 7 Spain.

$8 \quad 2$ Pompeu Fabra University, Barcelona, Catalonia, Spain.

$9{ }^{3}$ Consortium for Biomedical Research in Epidemiology and Public Health (CIBERESP), Madrid, 10 Spain.

$11{ }^{4}$ Institut Hospital del Mar d'InvestigacionsMèdiques-Parc de Salut Mar, Barcelona, Catalonia, 12 Spain.

13 5Learning Disabilities Unit (UTAE), Neuropediatrics Department, Hospital Sant Joan de Déu,

14 Universitat de Barcelona, Barcelona, Spain 


\section{Highlights}

17 - We estimated air pollution exposure during commuting by foot of 1,234 children

18 - $\mathrm{PM}_{2.5}$ and $\mathrm{BC}$ levels were linked with a diminished growth of superior working memory

19 - We observed an impairment in the attention function (not statistically significant)

20 - Policies for cleaner walking routes to school should be implemented

\section{Capsule:}

22 Despite the relatively short time spent commuting, the exposure to $\mathrm{PM}_{2.5}$ and $\mathrm{BC}$ from the home 23 to school commutes by foot of 1,234 schoolchildren was associated with a reduction in the growth 24 of cognitive functions.

25

26 Keywords: Commuting, superior working memory, inattentiveness, $\mathrm{PM}_{2.5}, \mathrm{BC}$. 


\section{Abstract}

28

29

30

31
BACKGROUND: A few studies have reported associations between the exposure to traffic-related air pollution at school and/or home and cognitive development. The impact on cognitive development of the exposure to air pollutants during commuting has not been explored.

OBJECTIVES: We aimed to assess the role of the exposure to traffic-related air pollutants during walking commute to school on cognitive development of schoolchildren.

METHODS: We performed a longitudinal study of children $(n=1,234)$ from 39 schools in Barcelona (Catalonia, Spain) who commuted by foot to school. The children completed four computerized tests during a 12-month follow-up to assess their developmental trajectories in superior working memory ( $d$ ' of the three-back numbers test) and inattentiveness (hit reaction time standard error of the Attention Network Test). Average particulate matter $\leq 2.5 \mu \mathrm{m}\left(\mathrm{PM}_{2.5}\right)$, Black Carbon (BC) and $\mathrm{NO}_{2}$ concentrations were estimated using Land Use Regression for the shortest walking route to school. Differences in cognitive growth were evaluated by linear mixed effects models with ageby-pollutant interaction terms.

RESULTS: An interquartile range increase in $\mathrm{PM}_{2.5}$ concentration in commuting routes decreased the annual growth of superior working memory by 5.4 (95\% $\mathrm{Cl}[-10.2,-0.6])$ points. Similarly, for BC the growth reduction was $4.6(95 \% \mathrm{Cl}[-9.0,-0.1])$ points. We also observed an increase in inattentiveness, but the association was not significant. The effects were independent to the exposure at home and at school.

CONCLUSIONS: Exposure to $\mathrm{PM}_{2.5}$ and $\mathrm{BC}$ from the commutes by foot was associated with a reduction in the growth of superior working memory. Efforts should be made to implement pedestrian school pathways through low traffic streets in order to increase security and minimise children's exposure to air pollutants. 


\section{Introduction}

52 Good air quality is required for human health and wellbeing, however more than $80 \%$ of people 53 living in urban areas are exposed to air quality levels that are above the World Health Organization 54 (WHO) standards (WHO, 2016). There is increasing evidence of the adverse effects of exposure to 55 air pollution on children's health (WHO, 2005), and air pollution has been recently considered as a 56 suspected cause of developmental neurotoxicity (Block et al., 2012). In previous research within 57 the framework of the BREATHE (Brain Development and Air Pollution Ultrafine Particles in School 58 Children) project (http://www.creal.cat/projectebreathe), long-term exposures during school time 59 to traffic-related air pollutants (TRAPs) were associated with a reduction in cognitive development 60 (Basagaña et al., 2016; Sunyer et al., 2015). Studies assessing the impact of air pollution on 61 cognitive function in children are limited and mainly focused on the exposure at home or school 62 (Suades-González et al., 2015). Nonetheless, no studies have assessed the impact on 63 neurodevelopment of the exposure to air pollutants during commuting time.

64

65

66

67

68

69

70

71

72

73

74

75

76

77

78

79

80

81

Children are highly exposed to TRAPs during commuting from home to school which often coincides with traffic pollution peaks (Nieuwenhuijsen et al., 2015). Some studies have found that although time spent during commuting might be short, the commuters receive a high proportion of their daily TRAP dose (De Nazelle et al., 2013; Dons et al., 2012) including children commuting to school (Buonanno et al., 2012; Nieuwenhuijsen et al., 2015; Rivas et al., 2016). In particular, from a BREATHE subpopulation of 45 children, Rivas et al. (2016) showed that while children on average spent $6 \%$ of the daytime in commuting, it accounted for about $20 \%$ of the daily integrated dose of Black Carbon (BC). Moreover, evidence from toxicological and experimental studies indicates that these short bursts of high pollutant intakes may have disproportionate health impacts (Michaels and Kleinman, 2000) and the health effects are expected to be much more important in children, who inhale a higher normalized dose of airborne particles compared to adults (Buonanno et al., 2011).

The specific health effects from the exposure to air pollutants during commuting remain largely unexplored. Liu et al. (2015) observed associations between the exposure to $\mathrm{PM}_{2.5}$ and heart rate variability in young healthy subjects. $\mathrm{PM}_{2.5}$ concentrations were dependent on the commuting mode, with the highest concentrations and cardiovascular effects observed in the walking mode. 
The time spent in vehicles during commuting (cars, public transportation, motorcycles or bicycles) has also been linked with an increase in the risk of myocardial infarction in adults (Peters et al.,

84

85

\section{Methods} 2004). schoolchildren.

\section{Participants} Barcelona, Spain.

Some cognitive functions, such as working memory or attention, develop substantially during childhood and adolescence (Tamnes et al., 2013). These capacities are shown to be critical for academic achievement (Shelton et al., 2010). This work aimed to study the effects of long-term exposure to TRAPs during home to school commuting by foot on cognitive development in primary

This study was framed within the ERC-Advanced Grant (FP7) BREATHE Project. All children without special needs (mental, motor or sensory impairment reported by the school) in grades 2 to 4 (7-10 y of age) from 39 schools in Barcelona (Catalonia, Spain) were invited to participate of which 2,897 (59\%) children agreed. Participating schools were similar to the remaining schools in Barcelona in terms of the neighborhood socioeconomic vulnerability index ( 0.46 versus 0.50 , Kruskal-Wallis test $p=0.57$ ) and $\mathrm{NO}_{2}$ levels ( 51.5 versus $50.9 \mu \mathrm{g} / \mathrm{m}^{3}$, Kruskal-Wallis test $p=0.72$ ). All parents or guardians signed the informed consent form approved by the Clinical Research Ethical Committee (No. 2010/41221/I) of the Institut Hospital del Mar d'Investigacions Mèdiques-Parc de Salut Mar,

\section{Air pollution exposure during commuting, at school and at home}

Data on the main mode of commuting to and from school and time spent during commuting was obtained from parents via questionnaires. Within the BREATHE participants, 1,234 children reported only walking as the main mode of transport. We focused on walkers since mode choice is a determinant of the exposure to air pollutants during commuting (De Nazelle et al., 2012; Kaur and Nieuwenhuijsen, 2009). Estimation of the exposure during commuting other than walking implies several assumptions (e.g. indoor/outdoor ratios for each transport mode) that might lead to differential misclassification. For those BREATHE participants who walked to school, we 
111 identified the shortest walking route to school based on street networks (network distance) by 112 using the network analyst extension from ArcGIS software v10.

114 We estimated the average concentration of $\mathrm{NO}_{2}$, particulate matter $\leq 2.5 \mu \mathrm{m}\left(\mathrm{PM}_{2.5}\right)$ and $\mathrm{BC}\left(\mathrm{PM} \mathrm{M}_{2.5}\right.$ 115 absorbance) in each route using the Land Use Regression (LUR) models developed within the

116 European Study of cohorts for Air Pollution Effects (ESCAPE) and described elsewhere (Eeftens et 117 al., 2012b). LUR models are based on simultaneous measurements of vehicle exhaust markers, 118 such as $\mathrm{NO}_{2}$, taken at many locations throughout an urban area during multiple periods that are 119 temporally corrected to obtain annual concentrations. Various parameters derived from 120 geographic information systems (GIS; e.g. traffic and roadway density, traffic volumes, truck 121 routes, population density) are used to predict the concentration at specific locations (Beelen et 122 al., 2013; Eeftens et al., 2012a).

We estimated the average concentration in the identified shortest route for each individual that reported commute by walking. Only the morning route (home to school) was considered, since these trips are more regular and they take place during the same time of the day across the schoolchildren. Moreover, concentrations of $\mathrm{NO}_{2}, \mathrm{PM}_{2.5}$ and $\mathrm{BC}\left(\mathrm{PM}_{2.5}\right.$ absorbance) at each participant's home address and at school during the time of the study were also estimated using the ESCAPE LUR models.

\section{Outcomes: Cognitive Development}

132 Cognitive development was assessed through 12-month change in working memory and attention, 133 as these functions grow steadily during pre-adolescence (Anderson, 2002; Rueda et al., 2005) and 134 are strongly related to intelligence and learning (Norman and Shallice, 1986). From January 2012 135 to March 2013, children were evaluated every three months over four repeated visits, throughout 136 two academic years, using computerized tests that took approximately 40 minutes to complete. 137 We used computerized n-back test for assessing working memory (Anderson, 2002) and the child 138 Attention Network Test (ANT) to characterize inattentiveness (Rueda et al., 2004). Groups of 1013920 children wearing ear protectors were assessed together and supervised by one trained 140 examiner per 3-4 children. For the $n$-back test, we examined different $n$-back loads (up to three141 back) and stimuli (colors, numbers, letters, and words). For analysis here, we selected the three142 back load for number stimuli as it showed a clear age-dependent slope in the four measurements 
and had little learning effect (Sunyer et al., 2015). The three-back test predicts general mental abilities as well as superior functions such as fluid intelligence (hereafter called superior working memory) (Shelton et al., 2010). All sets of n-back tests started with colors as a training phase to ensure the participant's understanding. The n-back parameter analyzed was $d$ prime (d'), a measure of detection subtracting the normalized false alarm rate from the hit rate: (Zhit rate Zfalse alarm rate) $\times 100$. A higher $d^{\prime}$ indicates more accurate test performance. Among the ANT measures, we chose the hit reaction time standard error (HRT-SE; standard error of reaction time for correct responses), which is a measure of response speed consistency throughout the test (Conners and Multi-Health Systems, 2000). Our choose of HRT-SE among all the ANT measurements was because it showed very little learning effect and the clearest growth during the 12-month study period. A higher HRT-SE indicates highly variable reactions related to inattentiveness.

\section{Contextual and individual covariates}

Socio-demographic factors included child age, sex, questionnaire-based parents' responses on parental education (primary or less/secondary/university) and employment (self employed/employed/unemployed), marital status (married/not married), family origin (child and at least one parent born in Spain/two parents or the child born out of Spain), and a neighborhood socioeconomic vulnerability index (based on level of education, unemployment, and occupation at the census tract) according to both the school and home address. Attention deficit hyperactivity disorder (ADHD) symptoms (ADHD/DSM-IV Scales, American Psychiatric Association 2002) were reported by teachers. Parents completed the Strengths and Difficulties Questionnaire (SDQ) on child behavioral problems (Goodman, 2001).

\section{Statistical analysis}

A total of 1,234 children with complete data (i.e., repeated outcome at least twice and individual data on maternal education, age and address) and walking as the unique mode of transport to commute to school were included. Because of the multilevel nature of the data (i.e., multiple visits for each child within schools), we used linear mixed effects models with the four repeated cognitive parameters as outcomes (one test at a time), exposure to air pollution during commuting as fixed effect predictor, and child and school as nested random effects. An interaction between age at each visit and the air pollutant concentration (reported as interquartile range (IQR) 
175 increase) was included to capture changes in 12-month progress in cognitive trajectory associated 176 with the exposure to air pollutants during commuting (e.g. a coefficient of -5 in working memory 177 for an IQR increase in $\mathrm{NO}_{2}$ exposure indicated that those exposed to $\mathrm{NO}_{2}$ concentrations at the 178 75th percentile had a mean decrease of 5 point in the 12-month working memory progress, as 179 compared with those exposed to $\mathrm{NO}_{2}$ concentrations at the 25th percentile). These models were 180 further adjusted for potential confounders identified a priori (age, sex, maternal education (an 181 indicator of individual socioeconomic status (SES)), and residential neighborhood socio-economic 182 status). Moreover, we also carried out models adjusting for commuting time, school and home air 183 pollution, by also including the interaction with age for these variables.

Sensitivity analyses were also conducted to assess effect modification by high-/low-air-pollution school, sex, maternal education, and ADHD to explore the potential residual confounding and susceptibility. We assessed the linearity of the relation between air pollution and cognitive tests stratifying the exposure by tertiles. Statistical significance was set at $p<0.05$. Stata 12 (StataCorp) statistical package was used to carry out the analyses.

\section{Results}

The description of cognitive outcomes, air pollutant's concentration estimates for commuting, school and home, and the time and distance for commuting are described in Table 1. Levels of pollutants during commuting were higher than those at home or school, although for $\mathrm{PM}_{2.5}$ the levels were very similar to those at home and slightly higher than at school. Concentrations of TRAPs during commuting were moderately to strongly correlated to mean levels at home (spearman coefficients between 0.61 and 0.69 ) and school (between 0.60 and 0.83; Table 2).

The study participants (only walkers) had an average age (SD) of 8.5 (0.9) years old and $50 \%$ were female (Table 3). The average (SD) distance between home and school was 561 (314) m. Table 3 also shows the characteristics of those children from the BREATHE project that used other modes of transport for commuting different than foot. There were differences in most of the socioeconomic variables as well as in TRAPs levels measured at school between the children who commuted on foot and the rest of the children. Walker's residential areas faced higher 
socioeconomic vulnerability and the proportion of parents with lower educational level and unemployed was higher than in the rest of the children.

Table 4 presents the associations between TRAP concentrations during commuting and 12-month change for the two cognitive parameters. All the coefficients were negative for superior working memory and positive for inattentiveness, indicating some impairments, which were statistically significant for the associations between $\mathrm{BC}$ and $\mathrm{PM}_{2.5}$ and superior working memory. These associations remained statistically significant after further adjustment for the time spent on commuting or levels of pollutants at home or school (Table 4). These results did not change notably after the adjustment was made for pollutant levels at both school and home (data not shown). Therefore children receiving a higher exposure during commuting from home to school had a reduced growth in cognitive parameters during a 1-year follow-up. For example, the annual growth of superior working memory for an IQR increase of $\mathrm{PM}_{2.5}$ concentration during commuting had decreased by $5.4(95 \% \mathrm{Cl}[-10.2,-0.6])$ points ( $p=0.02$; Figure 1). Similarly, an IQR increase of BC concentration implied a reduction in the growth of superior working memory of $4.6(95 \% \mathrm{Cl}[-$ $9.0,-0.1])$ points $(p=0,045))$. When the exposure was categorized into tertiles (Table S1), a linear trend was observed in all models for superior working memory, being statistically significant for the associations for $\mathrm{BC}$ and $\mathrm{PM}_{2.5}$ ( $\mathrm{p}$-value<0.05). Regarding inattentiveness, we did not observe any statistically significant linear trend, but the estimates for $\mathrm{PM}_{2.5}$ and $\mathrm{BC}$ on the $3^{\text {rd }}$ tertiles suggested that children exposed to high levels of TRAPS had greater inattentiveness.

In stratified analysis (Table 5), associations of TRAP concentrations and superior working memory were similar in different strata of children in terms of maternal education. The associations were stronger for males (statistically significant for $\mathrm{BC}$ and $\mathrm{PM}_{2.5}$ ) than females. Moreover, the negative associations were stronger (although not statistically significant) in children attending schools classified at the design phase as highly polluted for BC, but the contrary was observed for exposures to $\mathrm{PM}_{2.5}$ and $\mathrm{NO}_{2}$. Regarding $\mathrm{ADHD}$, the stratification did not result in a notable change in our observed associations for superior working memory. 


\section{Discussion}

234 We found adverse associations between the exposure to TRAPs during commuting from home to

235 school and superior working memory. We also found an increase in inattentiveness, although the

236 estimates were not statistically significant. Our observed associations appeared to be independent

237 from TRAPs levels at school and at home in the present analysis. However, the collinearity

238 between concentrations at school, home and commuting makes it difficult to disentangle the

239 effect of commuting exposure from the effect of school or home exposure, even though we fitted

240 the different micro-environments in the same model. Thus, we consider we could calculate a

241 fraction of the impact attributed only to commuting.

242

243 Until now, most epidemiological studies linking air pollution and children's mental development

244 have been based on levels of air pollutants at home or at the living area (Chiu et al., 2013; Guxens

245 et al., 2012; Harris et al., 2015; van Kempen et al., 2010). In a recent review, Suades-González et al.

246 (2015) identified 17 studies assessing the effect of air pollution on cognitive and psychomotor

247 development in children. Of these, 9 were based on prenatal exposures and 8 on postnatal

248 exposures at home (Calderón-Garcidueñas et al., 2015, 2012; Chiu et al., 2013), at school (Clark et

249 al., 2010; Perera et al., 2012; Sunyer et al., 2015) and at the living area (Abid et al., 2014; Jung et

250 al., 2013). To our knowledge, this is the first study reporting the potential impact of the exposure

251 to air pollution during commuting on neurodevelopment.

252

253 Children included in this analysis were participants in the BREATHE study based on which we have 254 recently reported that exposure to air pollution at school was associated with a slower cognitive 255 development (Basagaña et al., 2016; Sunyer et al., 2017, 2015). Moreover, a subsample of 48 256 BREATHE children filled in a time-activity diary while they were carrying personal BC monitors 257 during 48h. We estimated that while these children spent only $6 \%$ of their daily time on 258 commuting, they received $20 \%$ of their daily BC dose during commuting (Rivas et al., 2016). Such a 259 relatively large proportion of daily dose receiving during commuting could be attributed to 260 concurrence of commuting to school with road traffic rush hours and the close proximity to the 261 source, especially in urban areas (Rivas et al., 2016). Despite the time spent for commuting being 262 very short, the $\mathrm{BC}$ daily dose is comparable to the one received while being at home (35\% dose) or 263 at school (37\%). Therefore, it is likely to observe an independent effect of the exposure to air 
264 pollutants during commuting from school on neurodevelopment. The relative contribution of time

265 and dose during commuting in BREATHE children is in accordance to those reported in other

266 studies. For instance, in a study of 36 adult volunteers, also in Barcelona, travel activities

267 accounted for $6 \%$ of people's time and $24 \%$ of their daily inhaled $\mathrm{NO}_{2}$ dose (De Nazelle et al.,

268 2013). Similarly, Dons et al. (2012) found, when evaluating personal exposure of adults to BC, that

269 while only $6 \%$ of participants' time was spent in transport, this corresponded to $21 \%$ of their

270 personal exposure and $30 \%$ of the total inhaled dose. Other studies using activity-based models or

271 personal monitors also demonstrated that the transport activity can be responsible for quite a

272 large part of integrated personal exposure to pollutants (Dons et al., 2014). Then, although most

273 people spend a relatively small amount of their time traveling, journey-time exposures are

274 disproportionately large in proportion of total personal exposures to air pollution and,

275 consequently, the impact of the journey exposure on health is therefore potentially significant.

276 Exposure during commuting to traffic emissions has already been associated with increased risk of

277 serious adverse health effects such as myocardial infarction (Peters et al., 2004) as well as with an

278 increase in the heart rate variability (Liu et al., 2015). Moreover, the impact is expected to be

279 much more marked in children, who inhale a higher normalized dose of airborne particles

280 compared to adults, due to both their lung capacity and breathing rates (Buonanno et al., 2011).

281

282 Recent epidemiological and toxicological studies have shown that ambient air pollution exposure 283 may have neurotoxic effects particularly in children (Block et al., 2012; Grandjean and Landrigan, 284 2014). Potential biological mechanisms underlying such effects include disruption of the blood285 brain barrier, oxidative stress and neuroinflammation. Air pollutants can reach the brain directly 286 via olfactory epithelium or at systemic level via the blood-brain barrier, where microglia, in 287 particular, respond to this toxicants with chronic activation and inducing inflammation and 288 oxidative stress (Block et al., 2012). The findings of the present study were similar to our previous 289 study (Sunyer et al., 2015), where we found an association for superior working memory, which is 290 a good predictor of learning achievement (Shelton et al., 2010). The prefrontal cortex, which is 291 crucial for working memory (Braver et al., 2001), is still immature during childhood (Luna et al., 292 2001). Therefore working memory is a function susceptible to be affected by the exposure to 293 neurotoxicants during childhood (Boucher et al., 2009). As for inattentiveness, we observed an 294 impairment in the attention function (higher HRT-SE), although the associations were not 295 significant. This might be because, beyond its age-related development, inattentiveness shows 
daily fluctuations as it is more dependent on acute exposures, as we previously observed in Sunyer et al. (2017). On the other hand, working memory is more stable over time and therefore it is more robust for assessing the effect of low chronic exposures (Baddeley, 1992; Norman and Shallice, 1986). Children with ADHD symptoms were included in the analysis. The stratified analysis for ADHD (Table 5) did not result in a notable change in our observed associations for superior working memory. In previous research within BREATHE project we found that children with ADHD had a lower working memory at baseline, but a similar cognitive growth trajectories in the numbers variants of the n-back task, as compared to children without ADHD symptoms (LópezVicente et al., 2016). The sensitivity analysis (Table 5) indicated greater difficulties in focusing attention in children with ADHD symptoms (presence of more than 6 symptoms from the Criteria of Diagnostic and Statistical Manual of Mental Disorders list (ADHD-DSM-IV; American Psychiatric Association, 2002). Similar to our previous work (Sunyer et al., 2017), we found that traffic pollution is negatively associated with inattention irrespective of the presence or absence of this syndrome.

In the sex-stratified analyses, the association was restricted only to males who appeared to be much more susceptible to the exposure to $\mathrm{PM}_{2.5}, \mathrm{BC}$ and $\mathrm{NO}_{2}$ during commuting. For females, the effect is much lower and always not significant. This higher susceptibility for males was also observed for the exposure to BC (as Elemental Carbon) at school (Sunyer et al., 2015). These findings were consistent with those of animal studies showing that males were more susceptible to airborne metals than females, which might be because of sex-specific altered dopamine function (Curtis et al., 2010) or sex differences in oxidative stress (Cole et al., 2016).

Our study faced some limitations. Our exposure assessment was based on LUR models which have limited capability to capture the transient extreme peaks observed in commuting, especially at road crossings (Moreno et al., 2015). Likewise, the within-day variation is also not taken into account in the LUR models. Commuting very often takes place during traffic rush hours, which results in even higher exposures (De Nazelle et al., 2012; Rivas et al., 2017). Consequently, our exposure estimates for commuting may correspond to an underestimation of the actual concentrations which could have resulted in an underestimation of associations by our analyses. Therefore, our work is being conservative and we would expect even stronger associations if we had used real personal measurements. In order to have an accurate exposure assessment, the 
present study was only based on walkers since mode choice is thought to be the most influencing factor of exposure during travel (De Nazelle et al., 2012; Kaur and Nieuwenhuijsen, 2009). In case all children had been included, in-vehicle concentration in each transport mode should have been estimated (Smith et al., 2016). However, these concentrations are often estimated from several assumptions based on literature and probably wrongly extrapolated to all populations and places. Regarding to other factors influencing the exposure during commuting, such as timing of trips (time-of-day, day of the week), or time spent commuting, we selected only morning trips (from home to school). Morning trips are supposed to be more regular and they take place during the same time of the day (morning rush hours). Further adjustment of analyses for the time spent during commuting did not change our findings notably. An additional limitation is the potential residual confounding by socio-economic status (SES), because SES is associated with neurobehavioral outcomes in offspring (Hackman and Farah, 2009) and may also affect residential choice, school and mode of transport and thus determine the estimated exposures to ambient air pollution (Havard et al., 2009). We adjusted our analyses for indicators of SES at both individual (maternal education) and area (SES vulnerability index) levels. The stratified analyses did not reveal a considerable variation in the estimated associations across the strata of maternal education. Moreover, the socioeconomic characteristics appeared to be quite different between children participating in BREATHE according to the mode of transport.

Our findings could be wrongly interpreted as walking being detrimental for health. The benefits of active mobility are well established (Andersen et al., 2000; Council on Sports Medicine and Fitness and Council on School Health, 2006; Martínez-Gómez, 2011), however, it may also increase the exposure to air pollution, leading to negative health consequences. Nonetheless, walking or biking to school is a valuable opportunity for integrating physical activity into everyday life in children and most of the health impact studies conclude that benefits of active travel are significantly larger than the harm caused by increased pollutant inhalation (Mueller et al., 2015). Moreover, although the effects of the exposure in other modes of transport have not been assessed in the current study, it is known that people travelling by car or other passive modes are also highly exposed to air pollution (De Nazelle et al., 2012; Dons et al., 2012; Moreno et al., 2015; Zuurbier et al., 2010).

In conclusion, our study demonstrates that a short period of exposure to elevated concentrations 


\section{Acknowledgements}

367

368

369

370

371

372

373

374 and Khreis, 2016). contribution to the fieldwork.

\section{References} doi:10.1155/2014/628508 Mentales.

during commuting between home and school is associated with adverse impacts on cognitive development in schoolchildren. Efforts should be made to minimize children's exposures by increasing safer or "well-being" walking routes to school by reducing car use on school routes and thereby creating additional health benefits and safe environments for children (Nieuwenhuijsen

The research leading to these results has received funding from the European Community's Seventh Framework Program (ERC-Advanced Grant) under grant agreement number 268479 - the BREATHE project. Special thanks go to all the participant families and schools. We also acknowledge Cecilia Persavento, Judit González, Laura Bouso, and Pere Figueras for their

Abid, Z., Roy, A., Herbstman, J.B., Ettinger, A.S., 2014. Urinary polycyclic aromatic hydrocarbon metabolites and attention/deficit hyperactivity disorder, learning disability, and special education in U.S. children aged 6 to 15. J. Environ. Public Health 2014.

American Psychiatric Association, 2002. Manual Diagnóstico y Estadístico De Los Trastornos

Andersen, L.B., Schnohr, P., Schroll, M., Hein, H.O., 2000. All-Cause Mortality Associated With Physical Activity During Leisure Time, Work, Sports, and Cycling to Work 160, 1621-1628.

Anderson, P., 2002. Assessment and Development of Executive Function (EF) During Childhood. Child Neuropsychol. 8, 71-82. doi:10.1076/chin.8.2.71.8724

Baddeley, A., 1992. Working Memory. Science (80-. ). 255, 556-559.

Basagaña, X., Esnaola, M., Rivas, I., Amato, F., Alvarez-Pedrerol, M., Forns, J., López-Vicente, M., Pujol, J., Nieuwenhuijsen, M., Querol, X., Sunyer, J., 2016. Neurodevelopmental Deceleration by Urban Fine Particles from Different Emission Sources: A Longitudinal Observational Study. Environ. Health Perspect. 124, 1630-1636. doi:10.1289/EHP209 
Beelen, R., Hoek, G., Vienneau, D., Eeftens, M., Dimakopoulou, K., Pedeli, X., Tsai, M.-Y., Künzli, N., Schikowski, T., Marcon, A., Eriksen, K.T., Raaschou-Nielsen, O., Stephanou, E., Patelarou, E., Lanki, T., Yli-Tuomi, T., Declercq, C., Falq, G., Stempfelet, M., Birk, M., Cyrys, J., von Klot, S., Nádor, G., Varró, M.J., Dėdelè, A., Gražulevičienė, R., Mölter, A., Lindley, S., Madsen, C., Cesaroni, G., Ranzi, A., Badaloni, C., Hoffmann, B., Nonnemacher, M., Krämer, U., Kuhlbusch, T., Cirach, M., de Nazelle, A., Nieuwenhuijsen, M., Bellander, T., Korek, M., Olsson, D., Strömgren, M., Dons, E., Jerrett, M., Fischer, P., Wang, M., Brunekreef, B., de Hoogh, K., 2013. Development of NO2 and NOx land use regression models for estimating air pollution exposure in 36 study areas in Europe - The ESCAPE project. Atmos. Environ. 72, 10-23. doi:10.1016/j.atmosenv.2013.02.037

Block, M.L., Elder, A., Auten, R.L., Bilbo, S.D., Chen, H., Chen, J.-C., Cory-Slechta, D. a, Costa, D., Diaz-Sanchez, D., Dorman, D.C., Gold, D.R., Gray, K., Jeng, H.A., Kaufman, J.D., Kleinman, M.T., Kirshner, A., Lawler, C., Miller, D.S., Nadadur, S.S., Ritz, B., Semmens, E.O., Tonelli, L.H., Veronesi, B., Wright, R.O., Wright, R.J., 2012. The outdoor air pollution and brain health workshop. Neurotoxicology 33, 972-984. doi:10.1016/j.neuro.2012.08.014

Boucher, O., Muckle, G., Bastien, C.H., 2009. Prenatal exposure to polychlorinated biphenyls: A neuropsychologic analysis. Environ. Health Perspect. 117, 7-16. doi:10.1289/ehp.11294

Braver, T.S., Barch, D.M., Kelley, W.M., Buckner, R.L., Cohen, N.J., Miezin, F.M., Snyder, A.Z., Ollinger, J.M., Akbudak, E., Conturo, T.E., Petersen, S.E., 2001. Direct comparison of prefrontal cortex regions engaged by working and long-term memory tasks. Neuroimage 14, 48-59. doi:10.1006/nimg.2001.0791

Buonanno, G., Giovinco, G., Morawska, L., Stabile, L., 2011. Tracheobronchial and alveolar dose of submicrometer particles for different population age groups in Italy. Atmos. Environ. 45, 6216-6224. doi:10.1016/j.atmosenv.2011.07.066

Buonanno, G., Marini, S., Morawska, L., Fuoco, F.C., 2012. Individual dose and exposure of Italian children to ultrafine particles. Sci. Total Environ. 438, 271-7. doi:10.1016/j.scitotenv.2012.08.074

Calderón-Garcidueñas, L., Mora-Tiscareño, A., Franco-Lira, M., Zhu, H., Lu, Z., Solorio, E., TorresJardón, R., D'Angiulli, A., 2015. Decreases in short term memory, IQ, and altered brain metabolic ratios in urban apolipoprotein $\varepsilon 4$ children exposed to air pollution. J. aAzheimer's Dis. 757-770. doi:10.3233/JAD-142685

Calderón-Garcidueñas, L., Mora-Tiscareño, A., Styner, M., Zhu, H., Torres-Jardón, R., Carlos, E., 
Solorio-López, E., Medina-Cortina, H., Kavanaugh, M., D’Angiulli, A., 2012. White matter hyperintensities, systemic inflammation, brain growth, and cognitive functions in children exposed to air pollution. J. Alzheimer's Dis. 31, 183-191. doi:10.3233/JAD-2012-120610

Chiu, Y.H.M., Bellinger, D.C., Coull, B.A., Anderson, S., Barber, R., Wright, R.O., Wright, R.J., 2013. Associations between traffic-related black carbon exposure and attention in a prospective birth cohort of urban children. Environ. Health Perspect. 121, 859-864. doi:10.1289/ehp.1205940

Clark, N.A., Demers, P.A., Karr, C.J., Koehoorn, M., Lencar, C., Tamburic, L., Brauer, M., 2010. Effect of early life exposure to air pollution on development of childhood asthma. Environ. Health Perspect. 118, 284-290. doi:10.1289/ehp.0900916

Cole, T.B., Coburn, J., Dao, K., Roqué, P., Chang, Y.-C., Kalia, V., Guilarte, T.R., Dziedzic, J., Costa, L.G., 2016. Sex and genetic differences in the effects of acute diesel exhaust exposure on inflammation and oxidative stress in mouse brain. Toxicology 374, 1-9. doi:http://dx.doi.org/10.1016/j.tox.2016.11.010

Conners, C.K., Multi-Health Systems, 2000. Conners' Continuous Performance Test II. North Tonwanda (New York).

Council on Sports Medicine and Fitness, Council on School Health, 2006. Active Healthy Living: Prevention of Childhood Obesity Through Increased Physical Activity. Pediatrics 117, 183442. doi:10.1542/peds.2006-0472

Curtis, J.T., Hood, A.N., Chen, Y., Cobb, G.P., Wallace, D.R., 2010. Chronic metals ingestion by prairie voles produces sex-specific deficits in social behavior: An animal model of autism. Behav. Brain Res. 213, 42-49. doi:http://dx.doi.org/10.1016/j.bbr.2010.04.028

De Nazelle, A., Fruin, S., Westerdahl, D., Martinez, D., Ripoll, A., Kubesch, N., Nieuwenhuijsen, M., 2012. A travel mode comparison of commuters' exposures to air pollutants in Barcelona. Atmos. Environ. 59, 151-159. doi:10.1016/j.atmosenv.2012.05.013

De Nazelle, A., Seto, E., Donaire-gonzalez, D., Mendez, M., Matamala, J., Nieuwenhuijsen, M.J., Jerrett, M., 2013. Improving estimates of air pollution exposure through ubiquitous sensing technologies. Environ. Pollut. 92-99. doi:10.1016/j.envpol.2012.12.032.Improving

Dons, E., Int Panis, L., Van Poppel, M., Theunis, J., Wets, G., 2012. Personal exposure to Black Carbon in transport microenvironments. Atmos. Environ. 55, 392-398. doi:10.1016/j.atmosenv.2012.03.020

Dons, E., Kochan, B., Bellemans, T., Wets, G., Panis, L.I., 2014. Modeling Personal Exposure to Air 
Pollution with AB2C: Environmental Inequality. Procedia Comput. Sci. 32, 269-276. doi:10.1016/j.procs.2014.05.424

Eeftens, M., Beelen, R., de Hoogh, K., Bellander, T., Cesaroni, G., Cirach, M., Declercq, C., Dėdelè, A., Dons, E., de Nazelle, A., Dimakopoulou, K., Eriksen, K., Falq, G., Fischer, P., Galassi, C., Gražulevičienė, R., Heinrich, J., Hoffmann, B., Jerrett, M., Keidel, D., Korek, M., Lanki, T., Lindley, S., Madsen, C., Mölter, A., Nádor, G., Nieuwenhuijsen, M., Nonnemacher, M., Pedeli, X., Raaschou-Nielsen, O., Patelarou, E., Quass, U., Ranzi, A., Schindler, C., Stempfelet, M., Stephanou, E., Sugiri, D., Tsai, M.-Y., Yli-Tuomi, T., Varró, M.J., Vienneau, D., Klot, S. von, Wolf, K., Brunekreef, B., Hoek, G., 2012a. Development of Land Use Regression models for PM2.5, PM2.5 absorbance, PM10 and PMcoarse in 20 European study areas; results of the ESCAPE project. Environ. Sci. Technol. 46, 11195-205. doi:10.1021/es301948k

Eeftens, M., Tsai, M.-Y., Ampe, C., Anwander, B., Beelen, R., Bellander, T., Cesaroni, G., Cirach, M., Cyrys, J., de Hoogh, K., De Nazelle, A., de Vocht, F., Declercq, C., Dėdelè, A., Eriksen, K., Galassi, C., Gražulevičienè, R., Grivas, G., Heinrich, J., Hoffmann, B., lakovides, M., Ineichen, A., Katsouyanni, K., Korek, M., Krämer, U., Kuhlbusch, T., Lanki, T., Madsen, C., Meliefste, K., Mölter, A., Mosler, G., Nieuwenhuijsen, M., Oldenwening, M., Pennanen, A., Probst-Hensch, N., Quass, U., Raaschou-Nielsen, O., Ranzi, A., Stephanou, E., Sugiri, D., Udvardy, O., Vaskövi, É., Weinmayr, G., Brunekreef, B., Hoek, G., 2012b. Spatial variation of PM2.5, PM10, PM2.5 absorbance and PMcoarse concentrations between and within 20 European study areas and the relationship with NO2 - Results of the ESCAPE project. Atmos. Environ. 62, 303-317. doi:10.1016/j.atmosenv.2012.08.038

Goodman, R., 2001. Psychometric Properties of the Strengths and Difficulties Questionnaire. J. Am. Acad. Child Adolesc. Psychiatry 40, 1337-1345. doi:10.1097/00004583-200111000-00015

Grandjean, P., Landrigan, P.J., 2014. Neurobehavioural effects of developmental toxicity. Lancet Neurol. 13, 330-338. doi:10.1016/S1474-4422(13)70278-3

Guxens, M., Aguilera, I., Ballester, F., Estarlich, M., Fernández-Somoano, A., Lertxundi, A., Lertxundi, N., Mendez, M. a., Tardón, A., Vrijheid, M., Sunyer, J., 2012. Prenatal exposure to residential air pollution and infant mental development: Modulation by antioxidants and detoxification factors. Environ. Health Perspect. 120, 144-149. doi:org/10.1289/ehp.1103469 Hackman, D., Farah, M., 2009. Socioeconomic status and the developing brain. Trends Cogn. Sci. 13, 65-73. doi:10.1016/j.tics.2008.11.003.Socioeconomic 
Gryparis, A., Kloog, I., Koutrakis, P., Bellinger, D.C., White, R.F., Sagiv, S.K., Oken, E., 2015. Prenatal and childhood traffic-related pollution exposure and childhood cognition in the project viva cohort (Massachusetts, USA). Environ. Health Perspect. 123, 1072-1078. doi:10.1289/ehp.1408803

Havard, S., Deguen, S., Zmirou-Navier, D., Schillinger, C., Bard, D., 2009. Traffic-Related Air Pollution and Socioeconomic Status. Epidemiology 20, 223-230. doi:10.1097/EDE.0b013e31819464e1

Jung, C.-R., Lin, Y.-T., Hwang, B.-F., 2013. Air pollution and newly diagnostic autism spectrum disorders: a population-based cohort study in Taiwan. PLoS One 8, e75510. doi:10.1371/journal.pone.0075510

Kaur, S., Nieuwenhuijsen, M.J., 2009. Determinants of personal exposure to PM 2.5, ultrafine particle counts, and CO in a transport microenvironment. Environ. Sci. Technol. 43, 47374743. doi:10.1021/es803199z

Liu, W.-T., Ma, C.-M., Liu, I.-J., Han, B.-C., Chuang, H.-C., Chuang, K.-J., 2015. Effects of commuting mode on air pollution exposure and cardiovascular health among young adults in Taipei, Taiwan. Int. J. Hyg. Environ. Health 218, 319-323. doi:10.1016/j.ijheh.2015.01.003

López-Vicente, M., Forns, J., Suades-González, E., Esnaola, M., García-Esteban, R., ÁlvarezPedrerol, M., Júlvez, J., Burgaleta, M., Sebastián-Gallés, N., Sunyer, J., 2016. Developmental Trajectories in Primary Schoolchildren Using n-Back Task. Front. Psychol. 7, 1-11. doi:10.3389/fpsyg.2016.00716

Luna, B., Thulborn, K.R., Munoz, D.P., Merriam, E.P., Garver, K.E., Minshew, N.J., Keshavan, M.S., Genovese, C.R., Eddy, W.F., Sweeney, J.A., 2001. Maturation of widely distributed brain function subserves cognitive development. Neuroimage 13, 786-93. doi:10.1006/nimg.2000.0743

Martínez-Gómez, D., 2011. Active Commuting to School and Cognitive Performance in Adolescents. Arch. Pediatr. Adolesc. Med. 165, 300. doi:10.1001/archpediatrics.2010.244

Michaels, R. a., Kleinman, M.T., 2000. Incidence and Apparent Health Significance of Brief Airborne Particle Excursions. Aerosol Sci. Technol. 32, 93-105. doi:10.1080/027868200303803

Moreno, T., Reche, C., Rivas, I., Cruz Minguillón, M., Martins, V., Vargas, C., Buonanno, G., Parga, J., Pandolfi, M., Brines, M., Ealo, M., Sofia Fonseca, A., Amato, F., Sosa, G., Capdevila, M., de Miguel, E., Querol, X., Gibbons, W., 2015. Urban air quality comparison for bus, tram, subway and pedestrian commutes in Barcelona. Environ. Res. 142, 495-510. 
doi:10.1016/j.envres.2015.07.022

Mueller, N., Rojas-Rueda, D., Cole-Hunter, T., de Nazelle, A., Dons, E., Gerike, R., Götschi, T., Int Panis, L., Kahlmeier, S., Nieuwenhuijsen, M., 2015. Health impact assessment of active transportation: A systematic review. Prev. Med. (Baltim). 76, 103-114. doi:10.1016/j.ypmed.2015.04.010

Nieuwenhuijsen, M.J., Donaire-gonzalez, D., Rivas, I., Cirach, M., Hoek, G., Seto, E., Jerrett, M., Sunyer, J., 2015. Variability in and agreement between modelled and personal continuously measured black carbon levels using novel smartphone and sensor technologies. Environ. Sci. Technol. 49, 2977-2982. doi:10.1021/es505362x

Nieuwenhuijsen, M.J., Khreis, H., 2016. Car free cities: Pathway to healthy urban living. Environ. Int. 94, 251-262. doi:10.1016/j.envint.2016.05.032

Norman, D.A., Shallice, T., 1986. Attention to action: willed and automatic control of behavior, in: Davidson, R.J., Schwartz, G.E., Shapiro, D. (Eds.), Consciousnesss and Self-Regulation: Advances in Research and Theory. Vol.4. Plenum Press, New York, pp. 1-18.

Perera, F., Li, T.Y., Lin, C., Tang, D., 2012. Effects of prenatal polycyclic aromatic hydrocarbon exposure and environmental tobacco smoke on child IQ in a Chinese cohort. Environ. Res. 114, 40-46. doi:http://dx.doi.org/10.1016/j.envres.2011.12.011

Peters, A., Von Klot, S., Heier, M., Trentinaglia, I., Hörmann, A., Wichmann, E., Löwel, H., 2004. Exposure to Traffic and the Onset of Myocardial Infarction. N. Engl. J. Med. 351, 1721-1730. doi:10.1056/NEJMoa0904327

Rivas, I., Donaire-Gonzalez, D., Bouso, L., Esnaola, M., Pandolfi, M., de Castro, M., Viana, M., Àlvarez-Pedrerol, M., Nieuwenhuijsen, M., Alastuey, A., Sunyer, J., Querol, X., 2016. Spatiotemporally resolved black carbon concentration, schoolchildren's exposure and dose in Barcelona. Indoor Air 26, 391-402. doi:10.1111/ina.12214

Rivas, I., Kumar, P., Hagen-Zanker, A., 2017. Exposure to air pollutants during commuting in London: Are there inequalities among different socio-economic groups? Environ. Int. doi:10.1016/j.envint.2017.01.019

Rueda, M.R., Fan, J., McCandliss, B.D., Halparin, J.D., Gruber, D.B., Lercari, L.P., Posner, M.I., 2004. Development of attentional networks in childhood. Neuropsychologia 42, 1029-1040. doi:10.1016/j.neuropsychologia.2003.12.012

Rueda, M.R., Rothbart, M.K., McCandliss, B.D., Saccomanno, L., Posner, M.I., 2005. Training, maturation, and genetic influences on the development of executive attention. Proc. Natl. 
Acad. Sci. U. S. A. 102, 14931-6. doi:10.1073/pnas.0506897102

Shelton, J.T., Elliott, E.M., Matthews, R.A., Hill, B.D., Gouvier, W.D., 2010. The relationships of working memory, secondary memory, and general fluid intelligence: working memory is special. J. Exp. Psychol. Learn. Mem. Cogn. 36, 813-820. doi:10.1037/a0019046

Smith, J.D., Mitsakou, C., Kitwiroon, N., Barratt, B.M., Walton, H.A., Taylor, J.G., Anderson, H.R., Kelly, F.J., Beevers, S.D., 2016. London Hybrid Exposure Model: Improving Human Exposure Estimates to NO2 and PM2.5 in an Urban Setting. Environ. Sci. Technol. acs.est.6b01817. doi:10.1021/acs.est.6b01817

Suades-González, E., Gascon, M., Guxens, M., Sunyer, J., 2015. Air pollution and neuropsychological development: A review of the latest evidence. Endocrinology 156, 34733482. doi:10.1210/en.2015-1403

Sunyer, J., Esnaola, M., Alvarez-pedrerol, M., Forns, J., Rivas, I., Lopez-Vicente, M., SuadesGonzalez, E., Foraster, M., Garcia-Esteban, R., Basagaña, X., Viana, M., Cirach, M., Moreno, T., Alastuey, A., Sebastian-Galles, N., Nieuwenhuijsen, M., Querol, X., 2015. Association between Traffic-Related Air Pollution in Schools and Cognitive Development in Primary School Children: A Prospective Cohort Study. PLoS Med. 12, e1001792. doi:10.1371/journal.pmed.1001792

Sunyer, J., Suades-González, E., García-Esteban, R., Rivas, I., Pujol, J., Alvarez-Pedrerol, M., Forns, J., Querol, X., Basagaña, X., 2017. Traffic-related air pollution and attention in primary school children. Epidemiology 28, 181-189. doi:10.1097/EDE.0000000000000603

Tamnes, C.K., Walhovd, K.B., Grydeland, H., Holland, D., Østby, Y., Dale, A.M., Fjell, A.M., 2013. Longitudinal working memory development is related to structural maturation of frontal and parietal cortices. J. Cogn. Neurosci. 25, 1611-1623. doi:doi: 10.1162/jocn_a_00434

van Kempen, E., van Kamp, I., Lebret, E., Lammers, J., Emmen, H., Stansfeld, S., 2010. Neurobehavioral effects of transportation noise in primary schoolchildren: a cross-sectional study. Environ. Health 9, 25. doi:10.1186/1476-069X-9-25

WHO, 2016. Third WHO Urban Ambient Air Pollution Database.

WHO, 2005. Effects of Air Pollution on Children's Health and Development. A Review of the Evidence.

Zuurbier, M., Hoek, G., Oldenwening, M., Lenters, V., Meliefste, K., van den Hazel, P., Brunekreef, B., 2010. Commuters' exposure to particulate matter air pollution is affected by mode of transport, fuel type, and route. Environ. Health Perspect. 118, 783-9. 
doi:10.1289/ehp.0901622 
Table 1. Air pollutant concentration (estimated by LUR), time spent commuting and cognitive outcomes description (mean (standard deviation)). $\mathrm{N}=1,234$ (only walkers)

\begin{tabular}{|c|c|}
\hline \multicolumn{2}{|l|}{ Cognitive outcomes } \\
\hline \multicolumn{2}{|l|}{ Baseline (visit 1) } \\
\hline $\begin{array}{l}\text { 3-back Numbers, d' (Superior WM) } \\
\text { ANT, HRT-SE, milliseconds }\end{array}$ & $118(98)$ \\
\hline (inattentiveness) & $271(91)$ \\
\hline \multicolumn{2}{|l|}{ Change (visit 4 - visit 1) } \\
\hline 3-back Numbers, d' (Superior WM) & $21(132)$ \\
\hline $\begin{array}{l}\text { ANT, HRT-SE, milliseconds } \\
\text { (inattentiveness) }\end{array}$ & $-37(88)$ \\
\hline \multicolumn{2}{|l|}{ Air pollutants } \\
\hline \multicolumn{2}{|l|}{$\mathrm{PM}_{2.5}$} \\
\hline Commuting ( $\mu \mathrm{g} \mathrm{m}^{-3}$ ) & $17.5(2.7)$ \\
\hline Home $\left(\mu \mathrm{g} \mathrm{m}^{-3}\right)$ & $17.2(3.0)$ \\
\hline School ( $\left.\mu \mathrm{g} \mathrm{m}^{-3}\right)$ & $16.5(2.8)$ \\
\hline \multicolumn{2}{|l|}{ BC } \\
\hline Commuting ( $\mu \mathrm{g} \mathrm{m}^{-3}$ ) & $2.99(0.76)$ \\
\hline Home $\left(\mu \mathrm{g} \mathrm{m}^{-3}\right)$ & $2.84(0.84)$ \\
\hline School ( $\left.\mu \mathrm{g} \mathrm{m}^{-3}\right)$ & $2.54(0.66)$ \\
\hline \multicolumn{2}{|l|}{$\mathrm{NO}_{2}$} \\
\hline Commuting ( $\mu \mathrm{g} \mathrm{m}^{-3}$ ) & $83.0(32.3)$ \\
\hline Home $\left(\mu \mathrm{g} \mathrm{m}^{-3}\right)$ & $57.6(17.1)$ \\
\hline School $\left(\mu \mathrm{g} \mathrm{m}^{-3}\right)$ & $53.4(12.6)$ \\
\hline Time commuting (min) & $9.25(4.83)$ \\
\hline Distance (m) & $561(314)$ \\
\hline
\end{tabular}


Table 2. Spearman correlations of air pollutant concentration (commuting, home and school). All pollutants estimated by LUR. $\mathrm{N}=1,234$ (only walkers)

\begin{tabular}{|c|c|c|c|c|c|c|c|c|c|c|}
\hline & $\begin{array}{c}\mathrm{NO}_{2} \\
\text { comm }\end{array}$ & $\begin{array}{l}\mathrm{PM}_{2.5} \\
\text { comm }\end{array}$ & $\mathrm{BC}$ comm & $\begin{array}{c}\mathrm{NO}_{2} \\
\text { home }\end{array}$ & $\begin{array}{l}\mathrm{PM}_{2.5} \\
\text { home }\end{array}$ & BC home & $\begin{array}{c}\mathrm{NO}_{2} \\
\text { school }\end{array}$ & $\begin{array}{c}\mathrm{PM}_{2.5} \\
\text { school }\end{array}$ & BC school & $\begin{array}{l}\text { Time } \\
\text { comm }\end{array}$ \\
\hline $\mathrm{NO}_{2} \mathrm{comm}$ (LUR) & 1.00 & & & & & & & & & \\
\hline $\mathrm{PM}_{2.5}$ comm (LUR) & 0.84 & 1.00 & & & & & & & & \\
\hline BC comm (LUR) & 0.84 & 0.94 & 1.00 & & & & & & & \\
\hline $\mathrm{NO}_{2}$ home (LUR) & 0.67 & 0.64 & 0.64 & 1.00 & & & & & & \\
\hline $\mathrm{PM}_{2.5}$ home (LUR) & 0.66 & 0.69 & 0.62 & 0.81 & 1.00 & & & & & \\
\hline BC home (LUR) & 0.64 & 0.61 & 0.62 & 0.94 & 0.85 & 1.00 & & & & \\
\hline $\mathrm{NO}_{2}$ school (LUR) & 0.70 & 0.75 & 0.75 & 0.59 & 0.54 & 0.54 & 1.00 & & & \\
\hline $\mathrm{PM}_{2.5}$ school (LUR) & 0.75 & 0.83 & 0.78 & 0.60 & 0.58 & 0.56 & 0.90 & 1.00 & & \\
\hline BC school (LUR) & 0.60 & 0.65 & 0.68 & 0.54 & 0.48 & 0.49 & 0.95 & 0.82 & 1.00 & \\
\hline Time commuting & 0.03 & 0.08 & 0.05 & 0.00 & 0.00 & -0.03 & 0.01 & 0.02 & -0.01 & 1.00 \\
\hline
\end{tabular}

$\mathrm{NO}_{2}$ : Nitrogen Dioxide; $\mathrm{PM}_{2.5}$ : Particulate matter <2.5 $\mu \mathrm{m}$; BC: Black Carbon Carbon 
Table 4. Difference (and $95 \%$ confidence interval) ${ }^{\ddagger}$ in the 12 -month change, per commuting air pollution exposure and time spent commuting (interquartile range increase, IQR) in 1.234 children. Air pollution concentrations estimated by LUR.

\begin{tabular}{|c|c|c|c|c|}
\hline \multirow[b]{2}{*}{$\mathrm{PM}_{2.5}$ commuting } & \multicolumn{2}{|c|}{$\begin{array}{c}\text { Superior Working } \\
\text { Memory } \\
\text { (3-back Numbers, } \\
\text { d') }\end{array}$} & \multicolumn{2}{|c|}{$\begin{array}{l}\text { Inattentiveness } \\
\text { (ANT, HRT-SE, } \\
\text { milliseconds) }\end{array}$} \\
\hline & & & & \\
\hline Concentration & $-5.4^{*}$ & {$[-10.2,-0.6]$} & 2.3 & {$[-2.0,6.6]$} \\
\hline Concentration, adjusted for time & $-5.1^{*}$ & {$[-9.9,-0.4]$} & 2.3 & {$[-2.0,6.6]$} \\
\hline Concentration, adjusted for time and $\mathrm{PM}_{2.5}$ at school & $-9.0^{* *}$ & {$[-15.0,-3.1]$} & 3.2 & {$[-2.1,8.5]$} \\
\hline Concentration, adjusted for time and $\mathrm{PM}_{2.5}$ at home & $-8.2^{* *}$ & {$[-14.5,-1.8]$} & 3.1 & {$[-2.5,8.8]$} \\
\hline \multicolumn{5}{|l|}{$\mathrm{BC}$ commutting } \\
\hline Concentration & $-4.6^{*}$ & {$[-9.0,-0.1]$} & 2.2 & {$[-1.8,6.1]$} \\
\hline Concentration, adjusted for time & $-4.6^{*}$ & {$[-9.1,-0.2]$} & 2.2 & {$[-1.8,6.1]$} \\
\hline Concentration, adjusted for time and BC at school & $-7.8^{* *}$ & {$[-13.3,-2.3]$} & 2.9 & {$[-2.0,7.7]$} \\
\hline Concentration, adjusted for time and $\mathrm{BC}$ at home & $-7.3^{* *}$ & {$[-12.7,-1.9]$} & 3.5 & {$[-1.3,8.3]$} \\
\hline \multicolumn{5}{|l|}{$\mathrm{NO}_{2}$ commuting } \\
\hline Concentration & -2.7 & {$[-7.8,2.4]$} & 1.9 & {$[-2.6,6.4]$} \\
\hline Concentration, adjusted for time & -2.8 & {$[-7.9,2.3]$} & 1.9 & {$[-2.6,6.4]$} \\
\hline Concentration, adjusted for time and $\mathrm{NO}_{2}$ at school & -5.4 & {$[-11.7,1.0]$} & 2.6 & {$[-3.0,8.3]$} \\
\hline Concentration, adjusted for time and $\mathrm{NO}_{2}$ at home & -5.4 & {$[-11.7,1.0]$} & 2.6 & {$[-3.0,8.3]$} \\
\hline \multicolumn{5}{|c|}{$\begin{array}{l}\ddagger \text { Difference in the 12-month change adjusted for age, sex, maternal education and residential neighborhood socio- } \\
\text { economic status and other exposure when specified; school and subject as nested random effects. }\end{array}$} \\
\hline $\begin{array}{l}{ }^{*} p<0.0{ }^{* *} p<0.01{ }^{* *} p<0.001 \\
\text { ANT: Attention Network Test; HRT-SE: Hit Reaction Time Standard } \\
\text { matter }<2.5 \mu \mathrm{m} \text {; BC: Black Carbon Carbon }\end{array}$ & $2: \mathrm{N}$ & Dioxide; & & iculate \\
\hline
\end{tabular}


Table 5. Stratified analysis of TRAPs concentration during commuting (only walkers) from home to school (interquartile range increase) on the 12-month change of neurodevelopmental outcomes (superior working memory and inattentiveness). Adjusted models ${ }^{\pi}$

\begin{tabular}{|c|c|c|c|c|c|c|c|c|}
\hline & \multicolumn{2}{|c|}{$\begin{array}{l}\text { By School Air Pollution } \\
\text { (design) }\end{array}$} & \multicolumn{2}{|c|}{ By Maternal Education } & \multicolumn{2}{|c|}{ By Sex } & \multicolumn{2}{|c|}{ By ADHD ${ }^{\S}$} \\
\hline & $\begin{array}{l}\text { Low } \\
(n=369)\end{array}$ & $\begin{array}{l}\text { High } \\
(n=834)\end{array}$ & $\begin{array}{l}\text { Low } \\
(n=566)\end{array}$ & $\begin{array}{l}\text { High } \\
(n=637)\end{array}$ & $\begin{array}{l}\text { Male } \\
(n=602)\end{array}$ & $\begin{array}{l}\text { Female } \\
(n=601)\end{array}$ & $\begin{array}{c}\text { No } \\
(\mathrm{n}=1,099)\end{array}$ & $\begin{array}{c}\text { Yes } \\
(n=120)\end{array}$ \\
\hline \multicolumn{9}{|c|}{ Superior Working Memory (3-back Numbers, d') } \\
\hline $\mathrm{PM}_{2.5}$ & $\begin{array}{c}-4.4 \\
{[-12.8,4.0]}\end{array}$ & $\begin{array}{c}-2.0 \\
{[-9.6,5.6]}\end{array}$ & $\begin{array}{c}-6.2 \\
{[-12.6,0.2]}\end{array}$ & $\begin{array}{c}-5.1 \\
{[-12.3,2.0]}\end{array}$ & $\begin{array}{c}-9.2^{*} \\
{[-16.6,-1.8]}\end{array}$ & $\begin{array}{c}-2.1 \\
{[-8.3,4.0]}\end{array}$ & $\begin{array}{c}-6.0^{*} \\
{[-11.0,-1.0]}\end{array}$ & $\begin{array}{c}-4.9 \\
{[-20.2,10.4]}\end{array}$ \\
\hline BC & $\begin{array}{c}-1.4 \\
{[-10.4,7.5]}\end{array}$ & $\begin{array}{c}-2.2 \\
{[-9.1,4.8]}\end{array}$ & $\begin{array}{c}-4.5 \\
{[-10.6,1.5]}\end{array}$ & $\begin{array}{c}-4.8 \\
{[-11.5,1.8]}\end{array}$ & $\begin{array}{c}-9.7^{* *} \\
{[-16.8,-2.7]}\end{array}$ & $\begin{array}{c}-0.6 \\
{[-6.3,5.1]}\end{array}$ & $\begin{array}{c}-5.0^{*} \\
{[-9.8,-0.4]}\end{array}$ & $\begin{array}{c}-4.0 \\
{[-18.6,10.7]}\end{array}$ \\
\hline $\mathrm{NO}_{2}$ & $\begin{array}{c}-0.3 \\
{[-11.6,12.2]}\end{array}$ & $\begin{array}{c}0.8 \\
{[-6.0,7.7]}\end{array}$ & $\begin{array}{c}-3.1 \\
{[-10.0,3.7]}\end{array}$ & $\begin{array}{c}-2.7 \\
{[-10.3,4.8]}\end{array}$ & $\begin{array}{c}-6.5 \\
{[-14.2,1.3]}\end{array}$ & $\begin{array}{c}0.0 \\
{[-6.6,6.6]}\end{array}$ & $\begin{array}{c}-2.8 \\
{[-8.2,2.5]}\end{array}$ & $\begin{array}{c}-2.6 \\
{[-19.1,13.9]}\end{array}$ \\
\hline \multicolumn{9}{|c|}{ Inattentiveness (ANT, HRT-SE, milliseconds) } \\
\hline $\mathrm{PM}_{2.5}$ & $\begin{array}{c}-1.9 \\
{[-9.7,5.8]}\end{array}$ & $\begin{array}{c}2.4 \\
{[-4.3,9.0]}\end{array}$ & $\begin{array}{c}1.1 \\
{[-4.9,7.2]}\end{array}$ & $\begin{array}{c}4.2 \\
{[-2.0,10.3]}\end{array}$ & $\begin{array}{c}-0.2 \\
{[-6.7,6.2]}\end{array}$ & $\begin{array}{c}4.9 \\
{[-0.7,10.6]}\end{array}$ & $\begin{array}{c}2.1 \\
{[-2.4,6.6]}\end{array}$ & $\begin{array}{c}5.3 \\
{[-8.5,19.1]}\end{array}$ \\
\hline BC & $\begin{array}{c}-1.1 \\
{[-9.2,7.0]}\end{array}$ & $\begin{array}{c}1.3 \\
{[-4.8,7.4]}\end{array}$ & $\begin{array}{c}1.2 \\
{[-4.5,6.8]}\end{array}$ & $\begin{array}{c}3.5 \\
{[-2.1,9.1]}\end{array}$ & $\begin{array}{c}2.8 \\
{[-3.4,8.9]}\end{array}$ & $\begin{array}{c}2.1 \\
{[-3.1,7.3]}\end{array}$ & $\begin{array}{c}2.2 \\
{[-2.0,6.4]}\end{array}$ & $\begin{array}{c}3.7 \\
{[-9.5,16.9]}\end{array}$ \\
\hline $\mathrm{NO}_{2}$ & $\begin{array}{c}1.0 \\
{[-9.7,11.7]}\end{array}$ & $\begin{array}{c}-0.4 \\
{[-6.4,5.7]}\end{array}$ & $\begin{array}{c}2.6 \\
{[-3.8,9.0]}\end{array}$ & $\begin{array}{c}1.3 \\
{[-5.1,7.8]}\end{array}$ & $\begin{array}{c}3.2 \\
{[-3.6,10.0]}\end{array}$ & $\begin{array}{c}1.6 \\
{[-4.4,7.6]}\end{array}$ & $\begin{array}{c}1.8 \\
{[-2.9,6.6]}\end{array}$ & $\begin{array}{c}4.7 \\
{[-10.1,19.6]}\end{array}$ \\
\hline
\end{tabular}




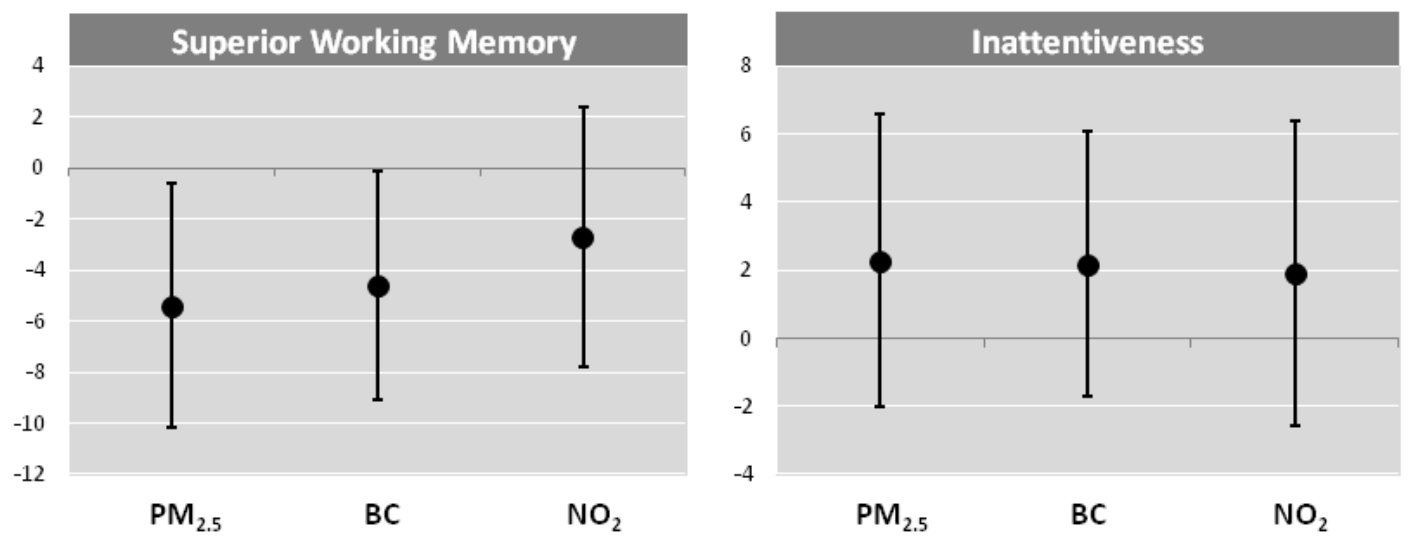

592 Figure 1. Change $(95 \% \mathrm{Cl})$ in cognitive growth per interquartile range increase of $\mathrm{PM}_{2.5}, \mathrm{BC}$ and $\mathrm{NO}_{2}$ 593 concentrations for children's commuting routes to school (only walkers). Models were adjusted for 594 for age, sex, maternal education and residential neighborhood socio-economic status; school and 595 subject as nested random effects. Superior working memory measured with 3-back numbers, $d^{\prime} \times$ 596 100. Inattentiveness measured with ANT, HRT-SE, ms. 


\title{
Impact of commuting exposure to traffic-related air pollution on cognitive development in children walking to school
}

\author{
Mar Alvarez-Pedrerol ${ }^{1,2,3}$, loar Rivas ${ }^{1,2,3}$, Mònica López-Vicente $^{1,2,3}$, Elisabet Suades- \\ González $z^{1,2,3,5}$, David Donaire-Gonzalez ${ }^{1,2,3}$, Marta Cirach ${ }^{1,2,3}$, Montserrat de Castro ${ }^{1,2,3}$, Mikel \\ Esnaola $^{1,2,3}$, Xavier Basagaña ${ }^{1,2,3}$, Payam Dadvand ${ }^{1,2,3}$, Mark Nieuwenhuijsen ${ }^{1,2,3}$, Jordi Sunyer \\ $1,2,3,4$. \\ ${ }^{1}$ ISGlobal, Centre for Research in Environmental Epidemiology (CREAL), Barcelona, Catalonia, \\ Spain. \\ ${ }^{2}$ Pompeu Fabra University, Barcelona, Catalonia, Spain. \\ ${ }^{3}$ Consortium for Biomedical Research in Epidemiology and Public Health (CIBERESP), Madrid, \\ Spain. \\ ${ }^{4}$ Institut Hospital del Mar d'InvestigacionsMèdiques-Parc de Salut Mar, Barcelona, Catalonia, \\ Spain. \\ ${ }^{5}$ Learning Disabilities Unit (UTAE), Neuropediatrics Department, Hospital Sant Joan de Déu, \\ Universitat de Barcelona, Barcelona, Spain
}

\section{Highlights}

- We estimated air pollution exposure during commuting by foot of 1,234 children

- $\quad \mathrm{PM}_{2.5}$ and $\mathrm{BC}$ levels were linked with a diminished growth of superior working memory

- We observed an impairment in the attention function (not statistically significant)

- Policies for cleaner walking routes to school should be implemented 


\title{
2 Impact of commuting-time exposure to traffic-related air pollution on cognitive development in children.
}

\author{
Mar Alvarez-Pedrerol, loar Rivas, Mònica López-Vicente, Elisabet Suades-González, David Donaire, \\ Marta Cirach, Montserrat de Castro, Mikel Esnaola, Xavier Basagaña, Payam Dadvand, Mark \\ Nieuwenhuijsen, Jordi Sunyer.
}

Table S1: Association between commuting concentration to tertiles of TRAPs (interquartile range increase) during commutting from home to school and neurodevelopmental outcomes. Adjusted modelsł.

Table S1. Association between tertiles of TRAPs (interquartile range increase) during commuting from home to school and neurodevelopmental outcomes. Adjusted modelsł

\begin{tabular}{|c|c|c|}
\hline & $\begin{array}{l}\text { Superior Working } \\
\text { Memory } \\
\text { (3-back Numbers, d') }\end{array}$ & $\begin{array}{c}\text { Inattentiveness } \\
\text { (ANT, HRT-SE, } \\
\text { milliseconds) } \\
\end{array}$ \\
\hline \multicolumn{3}{|l|}{$\mathrm{PM}_{2.5}$} \\
\hline \multirow{2}{*}{$2^{\text {nd }}$ tertile } & -5.3 & -2.9 \\
\hline & {$[-13.7,3.0]$} & {$[-9.3,3.6]$} \\
\hline \multirow{2}{*}{$3^{\text {rd }}$ tertile } & $-20.1^{* * *}$ & 5.6 \\
\hline & {$[-29.5,-10.8]$} & {$[-2.0,13.3]$} \\
\hline \multicolumn{3}{|l|}{$B C$} \\
\hline \multirow{2}{*}{$2^{\text {nd }}$ tertile } & -4.1 & -2.1 \\
\hline & {$[-12.6,4.4]$} & {$[-8.7,4.6]$} \\
\hline \multirow{2}{*}{$3^{\text {rd }}$ tertile } & $-19.3^{* * *}$ & 3.8 \\
\hline & {$[-28.8,-9.8]$} & {$[-4.0,11.5]$} \\
\hline \multicolumn{3}{|l|}{$\mathrm{NO}_{2}$} \\
\hline \multirow{2}{*}{$2^{\text {nd }}$ tertile } & -4.3 & -4.0 \\
\hline & {$[-12.8,4.1]$} & {$[-10.5,2.4]$} \\
\hline \multirow{2}{*}{$3^{\text {rd }}$ tertile } & -6.2 & -0.8 \\
\hline & {$[-15.7,3.3]$} & {$[-8.6,6.9]$} \\
\hline
\end{tabular}

${ }^{*} \mathrm{p}<0.05^{* *} \mathrm{p}<0.01{ }^{* * *} \mathrm{p}<0.001$

$\ddagger$ Difference in the 12-month change adjusted for age, sex, maternal education, residential neighborhood socio-economic status and air pollution at school*age; school and subject as nested random effects. ANT: Attention Network Test; HRT-SE: Hit Reaction Time Standard Error; $\mathrm{NO}_{2}$ : Nitrogen Dioxide; $\mathrm{PM}_{2.5}$ : Particulate matter $<2.5 \mu \mathrm{m}$; BC: Black Carbon Carbon

Linear trend $\mathrm{P}$-value $<0.05$ for $\mathrm{BC}$ and $\mathrm{PM}_{2.5}$ on superior working memory. 
1 Impact of commuting exposure to traffic-related air pollution on cognitive development in children walking to school

3 Mar Alvarez-Pedrerol ${ }^{1,2,3}$, loar Rivas ${ }^{1,2,3}$, Mònica López-Vicente ${ }^{1,2,3}$, Elisabet Suades-González ${ }^{1,2,3,5}$,

4 David Donaire-Gonzalez ${ }^{1,2,3}$, Marta Cirach ${ }^{1,2,3}$, Montserrat de Castro ${ }^{1,2,3}$, Mikel Esnaola ${ }^{1,2,3}$, Xavier

5 Basagaña $a^{1,2,3}$, Payam Dadvand ${ }^{1,2,3}$, Mark Nieuwenhuijsen ${ }^{1,2,3}$, Jordi Sunyer ${ }^{1,2,3,4}$.

$6{ }^{1}$ ISGlobal, Centre for Research in Environmental Epidemiology (CREAL), Barcelona, Catalonia, 7 Spain.

$82{ }^{2}$ Pompeu Fabra University, Barcelona, Catalonia, Spain.

$9{ }^{3}$ Consortium for Biomedical Research in Epidemiology and Public Health (CIBERESP), Madrid, 10 Spain.

$11{ }^{4}$ Institut Hospital del Mar d'Investigacions Mèdiques-Parc de Salut Mar, Barcelona, Catalonia, 12 Spain.

$13{ }^{5}$ Learning Disabilities Unit (UTAE), Neuropediatrics Department, Hospital Sant Joan de Déu, 14 Universitat de Barcelona, Barcelona, Spain

Corresponding author:

Mar Alvarez Pedrerol, PhD

Barcelona Institute for Global Health

Barcelona Biomedical Research Park (PRBB)

Doctor Aiguader, 88 |08003 Barcelona, Spain

Tel.+34 93 214.73.90 | mar.alvarez@isglobal.org 


\section{Highlights}

25 - We estimated air pollution exposure during commuting by foot of 1,234 children.

26 - $\mathrm{PM}_{2.5}$ and $\mathrm{BC}$ levels were associated with diminished growth of working memory.

27 - No significant associations were observed between $\mathrm{NO}_{2}$ levels and growth of working

28 memory, or between air pollution levels and development of inattentiveness.

29 - Our findings support policies for cleaner walking routes to school should.

\section{Capsule:}

31 Exposure to $\mathrm{PM}_{2.5}$ and $\mathrm{BC}$ during commuting between home and school commutes in a sample of

321,234 primary schoolchildren was associated with a reduction in the 12-month growth of cognitive 33 functions.

34

35 Keywords: Commuting, working memory, inattentiveness, $\mathrm{PM}_{2.5}, \mathrm{BC}$. 


\section{Abstract}

37 A few studies have found associations between the exposure to traffic-related air pollution at 38 school and/or home and cognitive development. The impact on cognitive development of the 39 exposure to air pollutants during commuting has not been explored. We aimed to assess the role 40 of the exposure to traffic-related air pollutants during walking commute to school on cognitive 41 development of children. We performed a longitudinal study of children ( $n=1,234$, aged 7-10 y)

42 from 39 schools in Barcelona (Catalonia, Spain) who commuted by foot to school. Children were 43 tested four times during a 12-month follow-up to characterize their developmental trajectories of 44 working memory ( $d^{\prime}$ of the three-back numbers test) and inattentiveness (hit reaction time 45 standard error of the Attention Network Test). Average particulate matter $\leq 2.5 \mu \mathrm{m}\left(\mathrm{PM}_{2.5}\right)$, Black 46 Carbon $(\mathrm{BC})$ and $\mathrm{NO}_{2}$ concentrations were estimated using Land Use Regression for the shortest 47 walking route to school. Differences in cognitive growth were evaluated by linear mixed effects 48 models with age-by-pollutant interaction terms. Exposure to $\mathrm{PM}_{2.5}$ and $\mathrm{BC}$ from the commutes by 49 foot was associated with a reduction in the growth of working memory (an interquartile range 50 increase in $\mathrm{PM}_{2.5}$ and $\mathrm{BC}$ concentrations decreased the annual growth of working memory by 5.4 51 (95\% Cl $[-10.2,-0.6])$ and $4.6(95 \% \mathrm{Cl}[-9.0,-0.1])$ points, respectively). The findings for $\mathrm{NO}_{2}$ were 52 not conclusive and none of the pollutants were associated with inattentiveness. Efforts should be 53 made to implement pedestrian school pathways through low traffic streets in order to increase 54 security and minimize children's exposure to air pollutants. 


\section{Introduction}

57 Good air quality is required for human health and wellbeing, however more than $80 \%$ of people residing in urban areas are exposed to air pollutant concentrations that are above the World Health Organization (WHO) standards (WHO, 2016). There is increasing evidence of the adverse effects of exposure to air pollution on children's health (WHO, 2005), and air pollution has been recently considered as a suspected cause of developmental neurotoxicity (Block et al., 2012). In previous research within the framework of the BREATHE (Brain Development and Air Pollution Ultrafine Particles in School Children) project (http://www.creal.cat/projectebreathe), long-term exposures during school time to traffic-related air pollutants (TRAPs) were associated with a reduction in cognitive development (Basagaña et al., 2016; Sunyer et al., 2015). Studies evaluating the impact of air pollution on cognitive function in children are limited and mainly focused on the exposure at home or school (Suades-González et al., 2015). Nonetheless, no studies have assessed the impact on cognitive development of the exposure to air pollutants during commuting time.

Children are highly exposed to TRAPs during commuting from home to school which often coincides with traffic pollution peaks (Nieuwenhuijsen et al., 2015). Some studies have found that although time spent during commuting might be short, the commuters receive a high proportion of their daily TRAP dose (De Nazelle et al., 2013; Dons et al., 2012) including children commuting to school (Buonanno et al., 2012; Nieuwenhuijsen et al., 2015; Rivas et al., 2016). In particular, from a BREATHE subpopulation of 45 children, Rivas et al. (2016) showed that while children on average spent $6 \%$ of the daytime in commuting, it accounted for about $20 \%$ of the daily integrated dose of Black Carbon (BC). Moreover, toxicological and experimental studies have evidenced that these short exposure to extremely high pollutant concentration may have disproportionate health impacts (Michaels and Kleinman, 2000). These health effects are expected to be especially important in children, who breathe in a higher normalized dose of airborne particles than adults (Buonanno et al., 2011).

The specific health effects from the exposure to air pollutants during commuting remain largely unexplored. Liu et al. (2015) observed associations between the exposure to $\mathrm{PM}_{2.5}$ and heart rate variability in young healthy subjects. $\mathrm{PM}_{2.5}$ concentrations were dependent on the commuting mode, with the highest concentrations and cardiovascular effects observed in the walking mode. 
87 The time spent in vehicles during commuting (cars, public transportation, motorcycles or bicycles)

88 has also been associated with an increase in the risk of myocardial infarction in adults (Peters et 89 al., 2004).

90 Some cognitive functions, such as working memory or attention, develop substantially during 91 childhood and adolescence (Tamnes et al., 2013). These capacities are shown to be critical for 92 academic achievement (Shelton et al., 2010). This work aimed to study the effects of long-term 93 exposure to TRAPs during home to school commuting by foot on cognitive development in primary 94 schoolchildren.

\section{Methods}

\section{Participants}

A total of 2,897 children in grades 2 to 4 (7-10 y of age) attending 39 different schools in Barcelona (Catalonia, Spain) agreed to participate in the ERC-Advanced Grant (FP7) BREATHE Project. From the total number of BREATHE participants, we included in this study 1,234 children reporting walking as the unique mode of transport to commute to school and having complete data. All parents or guardians signed the informed consent form approved by the Clinical Research Ethical Committee (No. 2010/41221/I) of the Institut Hospital del Mar d'Investigacions MèdiquesParc de Salut Mar, Barcelona, Spain. Further information regarding the BREATHE and schools participating in the BREATHE project can be found elsewhere (Sunyer et al., 2015).

\section{Air pollution exposure during commuting, at school and at home}

Information on the main mode of commuting to and from school and time spent during commuting was obtained from parents via questionnaires. Within the BREATHE participants, 1,234 children reported only walking as the main mode of transport. We focused on walkers since mode choice is a determinant of the exposure to air pollutants during commuting (De Nazelle et al.,

112 2012; Kaur and Nieuwenhuijsen, 2009). Estimation of the exposure during commuting other than 113 walking implies several assumptions (e.g. indoor/outdoor ratios for each transport mode) that 114 might lead to differential misclassification. For those BREATHE participants who walked to school,

115 we identified the shortest walking route to school based on street networks (network distance) by 116 using the network analyst extension from ArcGIS software v10. 
118 We estimated the average concentration of $\mathrm{NO}_{2}$, particulate matter $\leq 2.5 \mu \mathrm{m}\left(\mathrm{PM}_{2.5}\right)$ and $\mathrm{BC}\left(\mathrm{PM} \mathrm{M}_{2.5}\right.$ 119 absorbance) in each route using the Land Use Regression (LUR) models developed within the 120 ESCAPE Project and described elsewhere (Eeftens et al., 2012). The validity of the ESCAPE LUR 121 model for $\mathrm{PM}_{2.5}$ Absorbance (proxy for $\mathrm{BC}$ ) was previously evaluated by Nieuwenhuijsen et al.

122 (2015) against personal BC measurements performed in a subsample of 53 BREATHE children with 123 correlation coefficients of 0.59 and 0.68 for home and school, respectively. We did not have 124 enough statistical power to validate the model for the commutes with the BREATHE subsample. 125 We estimated the average concentration in the identified shortest route for each individual that 126 reported commute by walking. Only the morning route (home to school) was considered, since 127 these trips are more regular and they take place during the same time of the day across the 128 schoolchildren. Moreover, concentrations of $\mathrm{NO}_{2}, \mathrm{PM}_{2.5}$ and $\mathrm{BC}\left(\mathrm{PM}_{2.5}\right.$ absorbance) at every 129 participant's home address and at school during the study period were also estimated using the 130 ESCAPE LUR models. We also calculated the exposure taking into account the commuting 131 duration, as a factor of concentration*time.

\section{Cognitive Development: working memory and inattentiveness}

134 From January 2012 to March 2013, throughout two academic years, we evaluated the children's 135 cognitive development (change in working memory and attention) every three months (resulting 136 in four visits over a year) using computerized tests that took approximately 40 minutes to 137 complete. These functions develop until adulthood. We used the $n$-back test for assessing working 138 memory (Anderson, 2002) and the child Attention Network Test (ANT) to characterize 139 inattentiveness, including the three attention networks (alerting, orienting, and executive control) 140 (Rueda et al., 2004). We investigated the d prime (d', a measure of detection) from the three-back 141 load for number stimuli. We selected the 3-back since this parameter showed a normal 142 distribution while the 2-back task accuracy was truncated at $100 \%$ of correct responses and d' at 1433.91 score. The selection of the number stimulus was informed by its evident dependency on age 144 and limited learning effect compared to other stimuli (López-Vicente et al., 2016). The three-back 145 test evaluates general cognitive functioning as well as functions such as fluid intelligence (from 146 now on called working memory) (Shelton et al., 2010). Higher values of $d^{\prime}$ designate higher 147 accuracy in the execution of the test. From the ANT measures, we evaluated the reaction time (RT) 148 of the three attention networks and the hit reaction time standard error (HRT-SE; standard error 
of reaction time for correct responses), which is a measure of response speed consistency

150 throughout the test (Conners and Multi-Health Systems, 2000). A higher HRT-SE means greatly

151 variable reaction related to inattentiveness (Suades-González et al., 2017). These tests have been

152 reported to have acceptable internal consistency, reasonable factorial structure, and good

153 criterion validity and statistical dependencies for use in general population (Forns et al., 2014).

\section{Covariates}

156 Socio-demographic factors included child age, sex, parental education (primary or 157 less/secondary/university) and employment (self employed/employed/unemployed), marital 158 status (married/not married), family origin (child and at least one parent born in Spain/two 159 parents or the child born out of Spain), and a socioeconomic vulnerability index of the 160 neighborhood (derived from the level of education, unemployment, and occupation at the census 161 tract) for both the school and home address. Teachers reported children's Attention deficit 162 hyperactivity disorder (ADHD) symptoms (ADHD/DSM-IV Scales, American Psychiatric Association 163 2002).

165 Statistical analysis

166 Because of the multilevel nature of the data (i.e., children with repeated measures within schools), 167 we used linear mixed effects models with the cognitive parameters from the four repeated visits 168 as outcomes, exposure to air pollution during commuting as a fixed effect predictor, and child and 169 school as nested random effects. We evaluated the interaction between the air pollutant 170 concentration and the children's age at each visit to detect changes in the 12-month cognitive 171 trajectory associated with the exposure to air pollutants during commuting. The exposure was 172 reported as interquartile range (IQR) increase (e.g. a coefficient of -5 in working memory for an 173 IQR increase in $\mathrm{NO}_{2}$ exposure indicated that those exposed to $\mathrm{NO}_{2}$ concentrations at the 75th 174 percentile had a mean decrease of 5 point in the 12-month working memory progress, as 175 compared with those exposed to $\mathrm{NO}_{2}$ concentrations at the 25th percentile). These models were 176 additionally adjusted for age, sex, maternal education (an indicator of individual socioeconomic 177 status (SES)), and residential neighborhood socio-economic status. Moreover, we also carried out 178 models adjusting for commuting time, school and home air pollution, by also including the 179 interaction with age for these variables. 
181 Sensitivity analyses were performed to assess effect modification by school air pollution levels

182 (low/high, according to the classification in the design), sex, maternal education, and ADHD to 183 explore the potential residual confounding and susceptibility. We stratified the exposure by 184 tertiles to assess the linearity of the relationship between the outcomes of the cognitive tests and 185 the air pollution concentrations. Statistical significance was set at $p<0.05$. We used Stata 12 186 (StataCorp) to conduct the analyses. Moreover, we repeated the analysis using exposure 187 (concentration*time spent commuting) instead of concentration as fixed effect predictor.

Results

191 The description of cognitive outcomes, air pollutant's concentration estimates for commuting, 192 school and home, and the time and distance for commuting are described in Table 1. Levels of 193 pollutants during commuting were higher than those at home or school, although for $\mathrm{PM}_{2.5}$ the 194 levels were very similar to those at home and slightly higher than at school. Concentrations of 195 TRAPs during commuting were moderately to strongly correlated to mean levels at home 196 (spearman coefficients between 0.61 and 0.69) and school (between 0.60 and 0.83; Table 2).

197 The study participants (only walkers) had an average age (SD) of 8.5 (0.9) years old and 50\% were 198 female (Table 3). The average (SD) distance between home and school was 561 (314) m. Table 3 199 also shows the characteristics of those children from the BREATHE project that used other modes 200 of transport for commuting different than foot. There were differences in most of the 201 socioeconomic variables as well as in TRAPs levels measured at school between the children who 202 commuted on foot and the rest of the children. Walker's residential areas faced higher 203 socioeconomic vulnerability and the proportion of parents with lower educational level and unemployed was higher than in the rest of the children.

205

206

207

208

209

210
Table 4 presents the associations between TRAPs concentrations during commuting and 12-month change for the two cognitive parameters. We observed inverse associations for working memory and direct associations for inattentiveness, both consistent with cognitive impairment; however, the associations were statistically significant only between $\mathrm{BC}$ and $\mathrm{PM}_{2.5}$ and working memory. As presented in Table 4, an interquartile range increase in $\mathrm{PM}_{2.5}$ and $\mathrm{BC}$ concentrations were respectively associated with reduction of $-5.4(95 \% \mathrm{Cl}[-10.2,-0.6])$ and $-4.6(95 \% \mathrm{Cl}[-9.0,-0.1])$ 
211 points, equivalent to a decrease of $4.6 \%(95 \% \mathrm{Cl}[-8.6 \%,-0.5 \%])$ and $3.9 \%(95 \% \mathrm{Cl}[-7.6 \%,-0.1 \%])$ of

212 the 12-month growth in working memory. These associations remained statistically significant

213 after further adjustment for the time spent on commuting or levels of pollutants at home and/or

214 school (Table 4 and Table S1). The coefficients for home and school levels were not statistically

215 significant in the single and multi-pollutant models (Table S1). When the exposure was categorized

216 into tertiles (Table S2), a dose-response trend was observed in all exposures for working memory,

217 being statistically significant for the associations for $B C$ and $\mathrm{PM}_{2.5}$ ( $p$-value for trend<0.05).

218 Regarding inattentiveness, we did not observe any statistically significant linear trend, but the

219 estimates for $\mathrm{PM}_{2.5}$ and $\mathrm{BC}$ on the 3rd tertiles suggested that children exposed to high levels of

220 TRAPS had greater inattentiveness. We observed similar results for the three attentional networks

221 (data not shown) and general inattentiveness, and therefore we only presented here the results

222 for the HRT-SE.

223 When the exposure (concentration*time) during commuting was included in the model (Table S3)

224 rather than simply concentration, we also observed an impairment on the working memory which

225 was significant for the three pollutants under study $\left(\mathrm{PM}_{2.5}, \mathrm{BC}\right.$, and $\left.\mathrm{NO}_{2}\right)$. The effects (i.e. the

226 coefficients) were stronger when considering the exposure than the concentration in the single-

227 pollutant model (not adjusted for concentration at home and/or at school), while they were

228 weaker when comparing the coefficients of the multipollutant models. No significant associations

229 were observed for inattentiveness.

230 In stratified analysis (Table 5), associations of TRAP concentrations and working memory were

231 similar in different strata of children in terms of maternal education. The associations were

232 stronger for males (the coefficients remaining statistically significant for $\mathrm{BC}$ and $\mathrm{PM}_{2.5}$ in the group

233 of males) than females. Moreover, the negative associations were stronger (although not

234 statistically significant) in children attending schools classified at the design phase as highly

235 polluted for $\mathrm{BC}$, but the contrary was observed for exposures to $\mathrm{PM}_{2.5}$ and $\mathrm{NO}_{2}$. Regarding $\mathrm{ADHD}$,

236 the stratification did not result in a notable change in the associations for working memory.

\section{Discussion}

238 We found adverse associations between the levels of $\mathrm{BC}$ and $\mathrm{PM}_{2.5}$ during commuting between 239 home and school and working memory. The findings for $\mathrm{NO}_{2}$ and working memory, and for all the 
pollutants and inattentiveness were not conclusive. Our observed associations appeared to be independent from TRAPs levels at school and at home in the present analysis. However, the potential collinearity between concentrations at school, home and commuting made it difficult to disentangle the effect of commuting exposure from the effect of school or home exposure, although the models fitted with concentrations from the different micro-environments indicate that the contribution to the effect on cognition of the exposure during commuting has an important role. When exposure was considered (concentration*time), instead of concentration alone, an effect on working memory was also observed for $\mathrm{NO}_{2}, \mathrm{BC}$ and $\mathrm{PM}_{2.5}$

Until now, most epidemiological studies linking air pollution and children's mental development have been based on levels of air pollutants at home or at the living area (Chiu et al., 2013; Guxens et al., 2012; Harris et al., 2015; van Kempen et al., 2010). In a recent review, Suades-González et al. (2015) identified 17 studies evaluating the impact of air pollution on cognitive and psychomotor development in children. Of these, 9 were based on prenatal exposures and 8 on postnatal exposures at home (Calderón-Garcidueñas et al., 2015, 2012; Chiu et al., 2013), at school (Clark et al., 2010; Perera et al., 2012; Sunyer et al., 2015) and at the living area (Abid et al., 2014; Jung et al., 2013). To our knowledge, this is the first study reporting the potential impact of the exposure to air pollution during commuting on cognitive development.

Children included in this analysis were participants in the BREATHE study based on which we have recently showed that exposure to air pollution at school was associated with a slower cognitive development (Basagaña et al., 2016; Sunyer et al., 2017, 2015). Moreover, a subsample of 48 BREATHE children completed a diary reporting their activities while they were carrying personal BC monitors for $48 \mathrm{~h}$. We estimated that only $6 \%$ of children's daily time was spent on commuting. However, during this time they obtained the $20 \%$ of their daily BC dose during commuting (Rivas et al., 2016). Such a relatively large proportion of daily dose received during commuting could be attributed to commuting to school coinciding with road traffic peak hours and the closeness to the source, especially in urban areas (Rivas et al., 2016). Despite the time spent for commuting being very short, the $\mathrm{BC}$ daily dose is comparable to the one received while being at home ( $35 \%$ dose) or at school (37\%). Therefore, it is likely to observe an independent effect of the exposure to air pollutants during commuting from school on cognitive development. The relative contribution of time and dose during commuting in BREATHE children is in accordance to those reported from 
272 personal measurements by De Nazelle et al. (2013) and Dons et al. (2012). Other studies using

273 models based on activity patterns also pointed to transport activity as responsible of a substantial

274 part of integrated personal exposure to pollutants (Dons et al., 2014). Consequently, the impact of

275 the exposure during commuting on health is potentially significant. Peters et al. (2004) found an

276 association between the exposure during commuting to traffic emissions with increased risk of

277 myocardial infarction and Liu et al. (2015) with a decrease in the heart rate variability. The impact

278 is expected to be more important in children because of a smaller lung capacity and higher

279 breathing rates than adults (Buonanno et al., 2011).

280

281 Ambient air pollution may have neurotoxic effects, particularly in children (Block et al., 2012;

282 Grandjean and Landrigan, 2014). Potential biological mechanisms underlying such effects include

283 disruption of the blood-brain barrier, oxidative stress and neuroinflammation. Air pollutants can

284 directly reach the brain through the olfactory epithelium or at systemic level via the blood-brain

285 barrier, where microglia, in particular, respond to this toxicants with chronic activation and

286 inducing inflammation and oxidative stress (Block et al., 2012). The findings of the present study

287 were similar to our previous study (Sunyer et al., 2015), where we found an association for

288 working memory, which is a good predictor of learning achievement (Shelton et al., 2010). The

289 prefrontal cortex, which is crucial for working memory (Braver et al., 2001), is still immature during

290 childhood (Luna et al., 2001). Therefore working memory is a function susceptible to be affected

291 by the exposure to neurotoxicants during childhood (Boucher et al., 2009). As for inattentiveness,

292 we observed an impairment in the attention function (higher HRT-SE), although the associations

293 were not significant. This might be because, beyond its age-related development, inattentiveness

294 shows daily fluctuations as it is more dependent on acute exposures, as we previously observed in

295 Sunyer et al. (2017). On the other hand, working memory is more stable over time and therefore it

296 is more robust for assessing the effect of low chronic exposures (Baddeley, 1992; Norman and

297 Shallice, 1986). Children with ADHD symptoms were included in the analysis. The stratified

298 analysis for ADHD (Table 5) did not result in a notable change in our observed associations for

299 working memory. In previous research within BREATHE project we found that children with ADHD

300 had a lower working memory at baseline, but their cognitive growth trajectories in the numbers

301 variants of the $n$-back task were similar to children without ADHD symptoms (López-Vicente et al.,

302 2016). The sensitivity analysis (Table 5) indicated greater difficulties in focusing attention in

303 children with ADHD symptoms (presence of more than 6 symptoms from the ADHD-DSM-IV; 
American Psychiatric Association, 2002). Similar to our previous work (Sunyer et al., 2017), we found that traffic pollution is negatively associated with inattention independently of the presence or absence of this syndrome.

In the sex-stratified analyses, the association was restricted only to males who appeared to be much more susceptible to the exposure to $\mathrm{PM}_{2.5}, \mathrm{BC}$ and $\mathrm{NO}_{2}$ during commuting. For females, the effect is much lower and always not significant. This higher susceptibility for males was also observed for the exposure to BC (as Elemental Carbon) at school (Sunyer et al., 2015). These findings were consistent with observations in animal studies showing that males were more susceptible to airborne metals than females, which might be explained by sex-specific altered dopamine function (Curtis et al., 2010) or sex differences in oxidative stress (Cole et al., 2016).

Our study faced some limitations. The exposure assessment was based on LUR models which have limited capability to capture the transient extreme peaks observed in commuting, especially at road crossings (Moreno et al., 2015). Likewise, the within-day variation is also not taken into account in the LUR models since the estimations obtained correspond to an annual mean. Commuting very often takes place during traffic rush hours, which results in even higher exposures (De Nazelle et al., 2012; Rivas et al., 2017, 2016). Consequently, our exposure estimates for commuting may correspond to an underestimation of the actual concentrations which could have resulted in an underestimation of associations by our analyses. In order to have an accurate exposure assessment, the present study was only based on walkers since mode choice is believed to be the most influential determinant of exposure during travel (De Nazelle et al., 2012; Kaur and Nieuwenhuijsen, 2009). In case all children had been included, in-vehicle concentration in each transport mode should have been estimated (Smith et al., 2016). However, these concentrations are often estimated from several assumptions based on literature and probably wrongly extrapolated to all populations and places. Regarding to other factors influencing the exposure during commuting, such as timing of trips (time of the day, day of the week), or time spent commuting, we selected only morning trips (from home to school). Morning trips are supposed to be more regular and they take place during the same time of the day (morning rush hours). Further adjustment of analyses for the time spent during commuting did not change our findings notably. An additional limitation is the potential residual confounding by socio-economic status (SES), because SES is associated with neurobehavioral outcomes in offspring (Hackman and Farah, 
336 2009) and may also affect residential choice, school and mode of transport and thus determine 337 the estimated exposures to ambient air pollution (Havard et al., 2009). The models were adjusted

338 for individual (maternal education) and area (SES vulnerability index) indicators of SES levels. The 339 stratified analyses did not reveal a considerable variation in the estimated associations across the 340 strata of maternal education. Moreover, the socioeconomic characteristics appeared to be quite 341 different between children participating in BREATHE according to the mode of transport. Finally, 342 the possible collinearity between concentrations at school, home and commuting was also a 343 limitation. However, the effects observed for commuting seemed to be independent from school 344 and home levels, since the coefficients for commuting were significant and showed a stronger 345 association both in the single-pollutant and multi-pollutant models, while the coefficients for 346 school and home were not statistically significant in any of the models.

347 These findings should not be understood as a detrimental effect of walking for health. The benefits 348 of active mobility are well established (Andersen et al., 2000; Council on Sports Medicine and 349 Fitness and Council on School Health, 2006; Martínez-Gómez, 2011), however, it may also increase 350 the exposure to air pollution, leading to negative health consequences. Nonetheless, walking or 351 biking to school is a valuable opportunity for integrating physical activity into everyday life in 352 children and most of the health impact studies conclude that benefits of active travel are 353 significantly larger than the harm caused by increased pollutant inhalation (Mueller et al., 2015). 354 Moreover, although the effects of the exposure in other modes of transport have not been 355 assessed in the current study, it is known that people travelling by car or other passive modes are 356 also highly exposed to air pollution (De Nazelle et al., 2012; Dons et al., 2012; Moreno et al., 2015; 357 Zuurbier et al., 2010).

359 In conclusion, our study demonstrates that a short period of exposure to elevated concentrations 360 during commuting between home and school is associated with adverse impacts on cognitive 361 development in schoolchildren. Efforts should be made to minimize children's exposures by 362 increasing safer or "well-being" walking routes to school by reducing car use on school routes and 363 thereby creating additional health benefits and safe environments for children (Nieuwenhuijsen 364 and Khreis, 2016). 
366 Acknowledgements

367 The research leading to these results has received funding from the European Community's 368 Seventh Framework Program (ERC-Advanced Grant) under grant agreement number 268479 - the 369 BREATHE project. Special thanks go to all the participant families and schools. We also 370 acknowledge Cecilia Persavento, Judit González, Laura Bouso, and Pere Figueras for their 371 contribution to the fieldwork.

\section{References}

Abid, Z., Roy, A., Herbstman, J.B., Ettinger, A.S., 2014. Urinary polycyclic aromatic hydrocarbon metabolites and attention/deficit hyperactivity disorder, learning disability, and special education in U.S. children aged 6 to 15. J. Environ. Public Health 2014. doi:10.1155/2014/628508

American Psychiatric Association, 2002. Manual Diagnóstico y Estadístico De Los Trastornos Mentales.

Andersen, L.B., Schnohr, P., Schroll, M., Hein, H.O., 2000. All-Cause Mortality Associated With Physical Activity During Leisure Time, Work, Sports, and Cycling to Work 160, 1621-1628.

Anderson, P., 2002. Assessment and Development of Executive Function (EF) During Childhood. Child Neuropsychol. 8, 71-82. doi:10.1076/chin.8.2.71.8724

Baddeley, A., 1992. Working Memory. Science (80-. ). 255, 556-559.

Basagaña, X., Esnaola, M., Rivas, I., Amato, F., Alvarez-Pedrerol, M., Forns, J., López-Vicente, M., Pujol, J., Nieuwenhuijsen, M., Querol, X., Sunyer, J., 2016. Neurodevelopmental Deceleration by Urban Fine Particles from Different Emission Sources: A Longitudinal Observational Study. Environ. Health Perspect. 124, 1630-1636. doi:10.1289/EHP209

Block, M.L., Elder, A., Auten, R.L., Bilbo, S.D., Chen, H., Chen, J.-C., Cory-Slechta, D. a, Costa, D., Diaz-Sanchez, D., Dorman, D.C., Gold, D.R., Gray, K., Jeng, H.A., Kaufman, J.D., Kleinman, M.T., Kirshner, A., Lawler, C., Miller, D.S., Nadadur, S.S., Ritz, B., Semmens, E.O., Tonelli, L.H., Veronesi, B., Wright, R.O., Wright, R.J., 2012. The outdoor air pollution and brain health workshop. Neurotoxicology 33, 972-984. doi:10.1016/j.neuro.2012.08.014

Boucher, O., Muckle, G., Bastien, C.H., 2009. Prenatal exposure to polychlorinated biphenyls: A neuropsychologic analysis. Environ. Health Perspect. 117, 7-16. doi:10.1289/ehp.11294 
Braver, T.S., Barch, D.M., Kelley, W.M., Buckner, R.L., Cohen, N.J., Miezin, F.M., Snyder, A.Z., Ollinger, J.M., Akbudak, E., Conturo, T.E., Petersen, S.E., 2001. Direct comparison of prefrontal cortex regions engaged by working and long-term memory tasks. Neuroimage 14, 48-59. doi:10.1006/nimg.2001.0791

Buonanno, G., Giovinco, G., Morawska, L., Stabile, L., 2011. Tracheobronchial and alveolar dose of submicrometer particles for different population age groups in Italy. Atmos. Environ. 45, 6216-6224. doi:10.1016/j.atmosenv.2011.07.066

Buonanno, G., Marini, S., Morawska, L., Fuoco, F.C., 2012. Individual dose and exposure of Italian children to ultrafine particles. Sci. Total Environ. 438, 271-7. doi:10.1016/j.scitotenv.2012.08.074

Calderón-Garcidueñas, L., Mora-Tiscareño, A., Franco-Lira, M., Zhu, H., Lu, Z., Solorio, E., TorresJardón, R., D’Angiulli, A., 2015. Decreases in short term memory, IQ, and altered brain metabolic ratios in urban apolipoprotein $\varepsilon 4$ children exposed to air pollution. J. aAzheimer's Dis. 757-770. doi:10.3233/JAD-142685

Calderón-Garcidueñas, L., Mora-Tiscareño, A., Styner, M., Zhu, H., Torres-Jardón, R., Carlos, E., Solorio-López, E., Medina-Cortina, H., Kavanaugh, M., D’Angiulli, A., 2012. White matter hyperintensities, systemic inflammation, brain growth, and cognitive functions in children exposed to air pollution. J. Alzheimer's Dis. 31, 183-191. doi:10.3233/JAD-2012-120610

Chiu, Y.H.M., Bellinger, D.C., Coull, B.A., Anderson, S., Barber, R., Wright, R.O., Wright, R.J., 2013. Associations between traffic-related black carbon exposure and attention in a prospective birth cohort of urban children. Environ. Health Perspect. 121, 859-864. doi:10.1289/ehp.1205940

Clark, N.A., Demers, P.A., Karr, C.J., Koehoorn, M., Lencar, C., Tamburic, L., Brauer, M., 2010. Effect of early life exposure to air pollution on development of childhood asthma. Environ. Health Perspect. 118, 284-290. doi:10.1289/ehp.0900916

Cole, T.B., Coburn, J., Dao, K., Roqué, P., Chang, Y.-C., Kalia, V., Guilarte, T.R., Dziedzic, J., Costa, L.G., 2016. Sex and genetic differences in the effects of acute diesel exhaust exposure on inflammation and oxidative stress in mouse brain. Toxicology 374, 1-9. doi:http://dx.doi.org/10.1016/j.tox.2016.11.010

Conners, C.K., Multi-Health Systems, 2000. Conners' Continuous Performance Test II. North Tonwanda (New York).

Council on Sports Medicine and Fitness, Council on School Health, 2006. Active Healthy Living: 
Prevention of Childhood Obesity Through Increased Physical Activity. Pediatrics 117, 183442. doi:10.1542/peds.2006-0472

Curtis, J.T., Hood, A.N., Chen, Y., Cobb, G.P., Wallace, D.R., 2010. Chronic metals ingestion by prairie voles produces sex-specific deficits in social behavior: An animal model of autism. Behav. Brain Res. 213, 42-49. doi:http://dx.doi.org/10.1016/j.bbr.2010.04.028

De Nazelle, A., Fruin, S., Westerdahl, D., Martinez, D., Ripoll, A., Kubesch, N., Nieuwenhuijsen, M., 2012. A travel mode comparison of commuters' exposures to air pollutants in Barcelona. Atmos. Environ. 59, 151-159. doi:10.1016/j.atmosenv.2012.05.013

De Nazelle, A., Seto, E., Donaire-gonzalez, D., Mendez, M., Matamala, J., Nieuwenhuijsen, M.J., Jerrett, M., 2013. Improving estimates of air pollution exposure through ubiquitous sensing technologies. Environ. Pollut. 92-99. doi:10.1016/j.envpol.2012.12.032.Improving

Dons, E., Int Panis, L., Van Poppel, M., Theunis, J., Wets, G., 2012. Personal exposure to Black Carbon in transport microenvironments. Atmos. Environ. 55, 392-398. doi:10.1016/j.atmosenv.2012.03.020

Dons, E., Kochan, B., Bellemans, T., Wets, G., Panis, L.I., 2014. Modeling Personal Exposure to Air Pollution with AB2C: Environmental Inequality. Procedia Comput. Sci. 32, 269-276. doi:10.1016/j.procs.2014.05.424

Eeftens, M., Tsai, M.-Y., Ampe, C., Anwander, B., Beelen, R., Bellander, T., Cesaroni, G., Cirach, M., Cyrys, J., de Hoogh, K., De Nazelle, A., de Vocht, F., Declercq, C., Dèdelè, A., Eriksen, K., Galassi, C., Gražulevičienė, R., Grivas, G., Heinrich, J., Hoffmann, B., lakovides, M., Ineichen, A., Katsouyanni, K., Korek, M., Krämer, U., Kuhlbusch, T., Lanki, T., Madsen, C., Meliefste, K., Mölter, A., Mosler, G., Nieuwenhuijsen, M., Oldenwening, M., Pennanen, A., Probst-Hensch, N., Quass, U., Raaschou-Nielsen, O., Ranzi, A., Stephanou, E., Sugiri, D., Udvardy, O., Vaskövi, É., Weinmayr, G., Brunekreef, B., Hoek, G., 2012. Spatial variation of PM2.5, PM10, PM2.5 absorbance and PMcoarse concentrations between and within 20 European study areas and the relationship with NO2 - Results of the ESCAPE project. Atmos. Environ. 62, 303-317. doi:10.1016/j.atmosenv.2012.08.038

Forns, J., Esnaola, M., Lopez-Vicente, M., Suades-Gonzalez, E., Alvarez-Pedrerol, M., Júlvez, J., Sebastián-Gallés, N., Sunyer, J., 2014.The n-back test and the attentional network task as measures of child neuropsychological development in epidemiological studies. Neuropsychology. 28, 519-529. doi:10.1037/neu0000085

Grandjean, P., Landrigan, P.J., 2014. Neurobehavioural effects of developmental toxicity. Lancet 
Neurol. 13, 330-338. doi:10.1016/S1474-4422(13)70278-3

Guxens, M., Aguilera, I., Ballester, F., Estarlich, M., Fernández-Somoano, A., Lertxundi, A., Lertxundi, N., Mendez, M. a., Tardón, A., Vrijheid, M., Sunyer, J., 2012. Prenatal exposure to residential air pollution and infant mental development: Modulation by antioxidants and detoxification factors. Environ. Health Perspect. 120, 144-149. doi:org/10.1289/ehp.1103469

Hackman, D., Farah, M., 2009. Socioeconomic status and the developing brain. Trends Cogn. Sci. 13, 65-73. doi:10.1016/j.tics.2008.11.003.Socioeconomic

Harris, M.H., Gold, D.R., Rifas-Shiman, S.L., Melly, S.J., Zanobetti, A., Coull, B.A., Schwartz, J.D., Gryparis, A., Kloog, I., Koutrakis, P., Bellinger, D.C., White, R.F., Sagiv, S.K., Oken, E., 2015. Prenatal and childhood traffic-related pollution exposure and childhood cognition in the project viva cohort (Massachusetts, USA). Environ. Health Perspect. 123, 1072-1078. doi:10.1289/ehp.1408803

Havard, S., Deguen, S., Zmirou-Navier, D., Schillinger, C., Bard, D., 2009. Traffic-Related Air Pollution and Socioeconomic Status. Epidemiology 20, 223-230. doi:10.1097/EDE.0b013e31819464e1

Jung, C.-R., Lin, Y.-T., Hwang, B.-F., 2013. Air pollution and newly diagnostic autism spectrum disorders: a population-based cohort study in Taiwan. PLoS One 8, e75510. doi:10.1371/journal.pone.0075510

Kaur, S., Nieuwenhuijsen, M.J., 2009. Determinants of personal exposure to PM 2.5, ultrafine particle counts, and CO in a transport microenvironment. Environ. Sci. Technol. 43, 47374743. doi:10.1021/es803199z

Liu, W.-T., Ma, C.-M., Liu, I.-J., Han, B.-C., Chuang, H.-C., Chuang, K.-J., 2015. Effects of commuting mode on air pollution exposure and cardiovascular health among young adults in Taipei, Taiwan. Int. J. Hyg. Environ. Health 218, 319-323. doi:10.1016/j.ijheh.2015.01.003

López-Vicente, M., Forns, J., Suades-González, E., Esnaola, M., García-Esteban, R., ÁlvarezPedrerol, M., Júlvez, J., Burgaleta, M., Sebastián-Gallés, N., Sunyer, J., 2016. Developmental Trajectories in Primary Schoolchildren Using n-Back Task. Front. Psychol. 7, 1-11. doi:10.3389/fpsyg.2016.00716

Luna, B., Thulborn, K.R., Munoz, D.P., Merriam, E.P., Garver, K.E., Minshew, N.J., Keshavan, M.S., Genovese, C.R., Eddy, W.F., Sweeney, J.A., 2001. Maturation of widely distributed brain function subserves cognitive development. Neuroimage 13, 786-93. doi:10.1006/nimg.2000.0743 
Martínez-Gómez, D., 2011. Active Commuting to School and Cognitive Performance in Adolescents. Arch. Pediatr. Adolesc. Med. 165, 300. doi:10.1001/archpediatrics.2010.244

Michaels, R. a., Kleinman, M.T., 2000. Incidence and Apparent Health Significance of Brief Airborne Particle Excursions. Aerosol Sci. Technol. 32, 93-105. doi:10.1080/027868200303803

Moreno, T., Reche, C., Rivas, I., Cruz Minguillón, M., Martins, V., Vargas, C., Buonanno, G., Parga, J., Pandolfi, M., Brines, M., Ealo, M., Sofia Fonseca, A., Amato, F., Sosa, G., Capdevila, M., de Miguel, E., Querol, X., Gibbons, W., 2015. Urban air quality comparison for bus, tram, subway and pedestrian commutes in Barcelona. Environ. Res. 142, 495-510. doi:10.1016/j.envres.2015.07.022

Mueller, N., Rojas-Rueda, D., Cole-Hunter, T., de Nazelle, A., Dons, E., Gerike, R., Götschi, T., Int Panis, L., Kahlmeier, S., Nieuwenhuijsen, M., 2015. Health impact assessment of active transportation: A systematic review. Prev. Med. (Baltim). 76, 103-114. doi:10.1016/j.ypmed.2015.04.010

Nieuwenhuijsen, M.J., Donaire-gonzalez, D., Rivas, I., Cirach, M., Hoek, G., Seto, E., Jerrett, M., Sunyer, J., 2015. Variability in and agreement between modelled and personal continuously measured black carbon levels using novel smartphone and sensor technologies. Environ. Sci. Technol. 49, 2977-2982. doi:10.1021/es505362x

Nieuwenhuijsen, M.J., Khreis, H., 2016. Car free cities: Pathway to healthy urban living. Environ. Int. 94, 251-262. doi:10.1016/j.envint.2016.05.032

Norman, D.A., Shallice, T., 1986. Attention to action: willed and automatic control of behavior, in: Davidson, R.J., Schwartz, G.E., Shapiro, D. (Eds.), Consciousnesss and Self-Regulation: Advances in Research and Theory. Vol.4. Plenum Press, New York, pp. 1-18.

Perera, F., Li, T.Y., Lin, C., Tang, D., 2012. Effects of prenatal polycyclic aromatic hydrocarbon exposure and environmental tobacco smoke on child IQ in a Chinese cohort. Environ. Res. 114, 40-46. doi:http://dx.doi.org/10.1016/j.envres.2011.12.011

Peters, A., Von Klot, S., Heier, M., Trentinaglia, I., Hörmann, A., Wichmann, E., Löwel, H., 2004. Exposure to Traffic and the Onset of Myocardial Infarction. N. Engl. J. Med. 351, 1721-1730. doi:10.1056/NEJMoa0904327

Rivas, I., Donaire-Gonzalez, D., Bouso, L., Esnaola, M., Pandolfi, M., de Castro, M., Viana, M., Àlvarez-Pedrerol, M., Nieuwenhuijsen, M., Alastuey, A., Sunyer, J., Querol, X., 2016. Spatiotemporally resolved black carbon concentration, schoolchildren's exposure and dose in Barcelona. Indoor Air 26, 391-402. doi:10.1111/ina.12214 
Rivas, I., Kumar, P., Hagen-Zanker, A., 2017. Exposure to air pollutants during commuting in London: Are there inequalities among different socio-economic groups? Environ. Int. 101, 143-157. doi:10.1016/j.envint.2017.01.019

Rueda, M.R., Fan, J., McCandliss, B.D., Halparin, J.D., Gruber, D.B., Lercari, L.P., Posner, M.I., 2004. Development of attentional networks in childhood. Neuropsychologia 42, 1029-1040. doi:10.1016/j.neuropsychologia.2003.12.012

Shelton, J.T., Elliott, E.M., Matthews, R.A., Hill, B.D., Gouvier, W.D., 2010. The relationships of working memory, secondary memory, and general fluid intelligence: working memory is special. J. Exp. Psychol. Learn. Mem. Cogn. 36, 813-820. doi:10.1037/a0019046

Smith, J.D., Mitsakou, C., Kitwiroon, N., Barratt, B.M., Walton, H.A., Taylor, J.G., Anderson, H.R., Kelly, F.J., Beevers, S.D., 2016. London Hybrid Exposure Model: Improving Human Exposure Estimates to NO2 and PM2.5 in an Urban Setting. Environ. Sci. Technol. 50, 11760-11768. doi:10.1021/acs.est.6b01817

Suades-González, E., Forns, J., García-Esteban, R., López-Vicente, M., Esnaola, M., ÁlvarezPedrerol, M., Julvez, J., Cáceres, A., Basagaña, X., López-Sala, A., Sunyer, J., 2017. A Longitudinal Study on Attention Development in Primary School Children with and without Teacher-Reported Symptoms of ADHD. Front. Psychol. 8, 655. doi:10.3389/fpsyg.2017.00655

Suades-González, E., Gascon, M., Guxens, M., Sunyer, J., 2015. Air pollution and neuropsychological development: A review of the latest evidence. Endocrinology 156, 34733482. doi:10.1210/en.2015-1403

Sunyer, J., Esnaola, M., Alvarez-pedrerol, M., Forns, J., Rivas, I., Lopez-Vicente, M., SuadesGonzalez, E., Foraster, M., Garcia-Esteban, R., Basagaña, X., Viana, M., Cirach, M., Moreno, T., Alastuey, A., Sebastian-Galles, N., Nieuwenhuijsen, M., Querol, X., 2015. Association between Traffic-Related Air Pollution in Schools and Cognitive Development in Primary School Children: A Prospective Cohort Study. PLoS Med. 12, e1001792. doi:10.1371/journal.pmed.1001792

Sunyer, J., Suades-González, E., García-Esteban, R., Rivas, I., Pujol, J., Alvarez-Pedrerol, M., Forns, J., Querol, X., Basagaña, X., 2017. Traffic-related air pollution and attention in primary school children. Epidemiology 28, 181-189. doi:10.1097/EDE.0000000000000603

Tamnes, C.K., Walhovd, K.B., Grydeland, H., Holland, D., Østby, Y., Dale, A.M., Fjell, A.M., 2013. Longitudinal working memory development is related to structural maturation of frontal and parietal cortices. J. Cogn. Neurosci. 25, 1611-1623. doi:doi: 10.1162/jocn_a_00434 
556 van Kempen, E., van Kamp, I., Lebret, E., Lammers, J., Emmen, H., Stansfeld, S., 2010.

557 Neurobehavioral effects of transportation noise in primary schoolchildren: a cross-sectional

558 study. Environ. Heal. 9, 25. doi:10.1186/1476-069X-9-25

559 WHO, 2016. Third WHO Urban Ambient Air Pollution Database.

560 WHO, 2005. Effects of Air Pollution on Children's Health and Development. A Review of the

$561 \quad$ Evidence.

562 Zuurbier, M., Hoek, G., Oldenwening, M., Lenters, V., Meliefste, K., van den Hazel, P., Brunekreef,

563 B., 2010. Commuters' exposure to particulate matter air pollution is affected by mode of

564 transport, fuel type, and route. Environ. Health Perspect. 118, 783-9.

565 doi:10.1289/ehp.0901622

566 
Table 1. Air pollutant concentration (estimated by LUR), time spent commuting and cognitive outcomes description (mean (standard deviation)). $\mathrm{N}=1,234$ (only walkers)

\section{Cognitive outcomes}

Baseline (visit 1)

3-back Numbers, d' ( WM)

$118(98)$

ANT, HRT-SE, milliseconds

(inattentiveness)

\section{Change (visit 4 - visit 1)}

3-back Numbers, d' ( WM)

ANT, HRT-SE, milliseconds

(inattentiveness)

$271(91)$

$21(132)$

$-37(88)$

\section{Air pollutants}

$\mathrm{PM}_{2.5}$

Commuting ( $\mu \mathrm{g} \mathrm{m}^{-3}$ )

Home $\left(\mu \mathrm{g} \mathrm{m}^{-3}\right)$

$17.2(3.0)$

School $\left(\mu \mathrm{g} \mathrm{m}^{-3}\right)$

$16.5(2.8)$

BC

Commuting $\left(\mu \mathrm{g} \mathrm{m}^{-3}\right)$

$2.99(0.76)$

Home $\left(\mu \mathrm{g} \mathrm{m}^{-3}\right)$

$2.84(0.84)$

School $\left(\mu \mathrm{g} \mathrm{m}^{-3}\right)$

$2.54(0.66)$

$\mathrm{NO}_{2}$

Commuting ( $\mu \mathrm{g} \mathrm{m}^{-3}$ )

$83.0(32.3)$

Home $\left(\mu \mathrm{g} \mathrm{m}^{-3}\right)$

$57.6(17.1)$

School $\left(\mu \mathrm{g} \mathrm{m}^{-3}\right)$

$53.4(12.6)$

Time commuting (min)

$9.25(4.83)$

Distance (m)

$561(314)$

d': detectability; WM: Working Memory; ANT: Attention Network Test; $\mathrm{NO}_{2}$ :

Nitrogen Dioxide; $\mathrm{PM}_{2.5}$ : Particulate matter $<2.5 \mu \mathrm{m}$; BC: Black Carbon 
Table 2. Spearman correlations of air pollutant concentration (commuting, home and school). All pollutants estimated by LUR. $\mathrm{N}=1,234$ (only walkers)

\begin{tabular}{|c|c|c|c|c|c|c|c|c|c|c|}
\hline & $\begin{array}{c}\mathrm{NO}_{2} \\
\text { comm }\end{array}$ & $\begin{array}{l}\mathrm{PM}_{2.5} \\
\text { comm }\end{array}$ & BC comm & $\begin{array}{c}\mathrm{NO}_{2} \\
\text { home }\end{array}$ & $\begin{array}{l}\mathrm{PM}_{2.5} \\
\text { home }\end{array}$ & BC home & $\begin{array}{c}\mathrm{NO}_{2} \\
\text { school }\end{array}$ & $\begin{array}{l}\mathrm{PM}_{2.5} \\
\text { school }\end{array}$ & BC school & $\begin{array}{l}\text { Time } \\
\text { comm }\end{array}$ \\
\hline $\mathrm{NO}_{2} \mathrm{comm}$ & 1.00 & & & & & & & & & \\
\hline $\mathrm{PM}_{2.5}$ comm & 0.84 & 1.00 & & & & & & & & \\
\hline BC comm & 0.84 & 0.94 & 1.00 & & & & & & & \\
\hline $\mathrm{NO}_{2}$ home & 0.67 & 0.64 & 0.64 & 1.00 & & & & & & \\
\hline $\mathrm{PM}_{2.5}$ home & 0.66 & 0.69 & 0.62 & 0.81 & 1.00 & & & & & \\
\hline BC home & 0.64 & 0.61 & 0.62 & 0.94 & 0.85 & 1.00 & & & & \\
\hline $\mathrm{NO}_{2}$ school & 0.70 & 0.75 & 0.75 & 0.59 & 0.54 & 0.54 & 1.00 & & & \\
\hline $\mathrm{PM}_{2.5}$ school & 0.75 & 0.83 & 0.78 & 0.60 & 0.58 & 0.56 & 0.90 & 1.00 & & \\
\hline BC school & 0.60 & 0.65 & 0.68 & 0.54 & 0.48 & 0.49 & 0.95 & 0.82 & 1.00 & \\
\hline Time commuting & 0.03 & 0.08 & 0.05 & 0.00 & 0.00 & -0.03 & 0.01 & 0.02 & -0.01 & 1.00 \\
\hline
\end{tabular}

$\mathrm{NO}_{2}$ : Nitrogen Dioxide; $\mathrm{PM}_{2.5}$ : Particulate matter $<2.5 \mu \mathrm{m} ; \mathrm{BC}$ : Black Carbon

All coefficients are statistically significant at $p<0.01$, except those correlations with time. 
Table 3. Description (percent or mean (standard deviation)) of the population (and school) characteristics by mode of transport used from home to school.

\begin{tabular}{|c|c|c|c|}
\hline & Foot & Others & $p$-value ${ }^{\ddagger}$ \\
\hline Number of children & 1234 & 1681 & \\
\hline \multicolumn{4}{|l|}{ Socio-economic variables } \\
\hline Age (y) & $8.5(0.9)$ & $8.5(0.9)$ & 0.400 \\
\hline Girls (\%) & 50 & 49 & 0.755 \\
\hline Foreign origin (\% non-Spanish) & 18 & 14 & 0.002 \\
\hline Siblings, no (\%) & 22 & 20 & 0.279 \\
\hline Adopted child (\%) & 3 & 4 & 0.423 \\
\hline Maternal education, university (\%) & 53 & 64 & $<0.001$ \\
\hline Paternal education, university (\%) & 45 & 60 & $<0.001$ \\
\hline Maternal occupation, unemployed (\%) & 21 & 12 & $<0.001$ \\
\hline Paternal occupation, unemployed (\%) & 14 & 5 & $<0.001$ \\
\hline Marital status, married (\%) & 85 & 86 & 0.552 \\
\hline Home SES vulnerability index & $0.48(0.19)$ & $0.42(0.22)$ & $<0.001$ \\
\hline Type of school, public (\%) & 41 & 32 & $<0.001$ \\
\hline \multicolumn{4}{|l|}{ Health \& life style } \\
\hline $\operatorname{ADHD}^{q}(\%)$ & 10 & 11 & 0.475 \\
\hline Overweight/obese (\%) & 30 & 26 & 0.017 \\
\hline Computer games weekend, $\geq 2 \mathrm{~h}(\%)$ & 34 & 29 & 0.012 \\
\hline Smoking during pregnancy (\%) & 10 & 10 & 0.594 \\
\hline Gestational age, < 37 weeks (\%) & 7 & 8 & 0.265 \\
\hline Birth weight < 2500 (\%) & 9 & 10 & 0.178 \\
\hline Breastfeeding, no (\%) & 16 & 20 & $<0.001$ \\
\hline \multicolumn{4}{|l|}{ Commuting } \\
\hline Distance from home to school (m) & $561(314)$ & $3001(2413)$ & $<0.001$ \\
\hline Time (min) & $9.2(4.8)$ & $15.5(10.7)$ & $<0.001$ \\
\hline $\mathrm{PM}_{2.5}\left(\mu \mathrm{g} \mathrm{m}^{-3}\right)$ & $17.5(2.7)$ & $17.4(2.8)$ & 0.917 \\
\hline $\mathrm{BC}\left(\mu \mathrm{g} \mathrm{m}^{-3}\right)$ & $2.99(0.76)$ & $2.74(0.67)$ & $<0.001$ \\
\hline $\mathrm{NO}_{2},\left(\mu \mathrm{g} \mathrm{m}^{-3}\right)$ & $83.0(32.3)$ & $76.3(27.9)$ & $<0.001$ \\
\hline \multicolumn{4}{|l|}{ School } \\
\hline $\mathrm{PM}_{2.5}\left(\mu \mathrm{g} \mathrm{m}^{-3}\right)$ & $16.5(2.8)$ & $14.8(3.6)$ & $<0.001$ \\
\hline $\mathrm{BC}\left(\mu \mathrm{g} \mathrm{m}^{-3}\right)$ & $2.54(0.66)$ & $1.82(0.89)$ & $<0.001$ \\
\hline $\mathrm{NO}_{2},\left(\mu \mathrm{g} \mathrm{m}^{-3}\right)$ & $53.4(12.6)$ & $38.9(17.8)$ & $<0.001$ \\
\hline
\end{tabular}

$\mathrm{PM}_{2.5}$ : Particulate matter <2.5 $\mu \mathrm{m}$; BC: Black Carbon; $\mathrm{NO}_{2}$ : Nitrogen Dioxide; ADHD: Attention Deficit Hyperactivity Disorder based on the DSMIV criteria; SES: socio-economic status based on the neighborhood vulnerability index;

$\S$ Includes: multi modal commuting (2 different modes per week), and commutes by tram. Statistics for those children commuting by 3 or modes of transports per week or commuting by train or underground are not shown.

${ }^{\ddagger}$ Kruskal-Wallis and Chi-square tests

"ADHD: children with more than 6 symptoms from the Criteria of Diagnostic and Statistical Manual of Mental Disorders list (ADHD-DSM-IV). 
Table 4. Difference (and $95 \%$ confidence interval) ${ }^{\ddagger}$ in the 12 -month change, per commuting air pollution concentration and time spent commuting (interquartile range increase, IQR) in 1.234 children. Air pollution concentrations estimated by LUR.

\begin{tabular}{|c|c|c|c|c|}
\hline \multirow{2}{*}{$\mathrm{PM}_{2.5}$ commuting } & \multicolumn{2}{|c|}{$\begin{array}{c}\text { Working Memory } \\
\text { (3-back Numbers, } \\
d^{\prime} \text { ) }\end{array}$} & \multicolumn{2}{|c|}{$\begin{array}{c}\text { Inattentiveness } \\
\text { (ANT, HRT-SE, } \\
\text { milliseconds) }\end{array}$} \\
\hline & & & & \\
\hline Concentration & $-5.4 *$ & {$[-10.2,-0.6]$} & 2.3 & {$[-2.0,6.6]$} \\
\hline Concentration, adjusted for time & $-5.1^{*}$ & {$[-9.9,-0.4]$} & 2.3 & {$[-2.0,6.6]$} \\
\hline Concentration, adjusted for time and $\mathrm{PM}_{2.5}$ at school & $-9.0 * *$ & {$[-15.0,-3.1]$} & 3.2 & {$[-2.1,8.5]$} \\
\hline Concentration, adjusted for time and $\mathrm{PM}_{2.5}$ at home & $-8.2^{* *}$ & {$[-14.5,-1.8]$} & 3.1 & {$[-2.5,8.8]$} \\
\hline $\begin{array}{l}\text { Concentration, adjusted for time and } \mathrm{PM}_{2.5} \text { at home \& } \\
\text { school }\end{array}$ & $-9.0 * *$ & {$[-15.4,-2.6]$} & 3.3 & {$[-2.4,9.0]$} \\
\hline \multicolumn{5}{|l|}{ BC commuting } \\
\hline Concentration & $-4.6^{*}$ & {$[-9.0,-0.1]$} & 2.2 & {$[-1.8,6.1]$} \\
\hline Concentration, adjusted for time & $-4.6^{*}$ & {$[-9.1,-0.2]$} & 2.2 & {$[-1.8,6.1]$} \\
\hline Concentration, adjusted for time and $\mathrm{BC}$ at school & $-7.8^{* *}$ & {$[-13.3,-2.3]$} & 2.9 & {$[-2.0,7.7]$} \\
\hline Concentration, adjusted for time and $\mathrm{BC}$ at home & $-7.3^{* *}$ & {$[-12.7,-1.9]$} & 3.5 & {$[-1.3,8.3]$} \\
\hline Concentration, adjusted for time and BC at home \& school & $-7.8^{* *}$ & {$[-13.4,-2.3]$} & 3.1 & {$[-1.8,8.0]$} \\
\hline \multicolumn{5}{|l|}{$\mathrm{NO}_{2}$ commuting } \\
\hline Concentration & -2.7 & {$[-7.8,2.4]$} & 1.9 & {$[-2.6,6.4]$} \\
\hline Concentration, adjusted for time & -2.8 & {$[-7.9,2.3]$} & 1.9 & {$[-2.6,6.4]$} \\
\hline Concentration, adjusted for time and $\mathrm{NO}_{2}$ at school & -5.4 & {$[-11.7,1.0]$} & 2.6 & {$[-3.0,8.3]$} \\
\hline Concentration, adjusted for time and $\mathrm{NO}_{2}$ at home & -5.4 & {$[-11.7,1.0]$} & 2.6 & {$[-3.0,8.3]$} \\
\hline Concentration, adjusted for time and $\mathrm{NO}_{2}$ at home $\&$ school & -5.4 & {$[-11.7,1.0]$} & 2.6 & {$[-3.0,8.3]$} \\
\hline \multicolumn{5}{|c|}{$\begin{array}{l}F \text { Difference in the 12-month change adjusted for age, sex, maternal education and residential neighborhood socio- } \\
\text { economic status and other exposure when specified; school and subject as nested random effects. }\end{array}$} \\
\hline \multicolumn{5}{|c|}{$\begin{array}{l}* p<0.05 * * p<0.01 \\
\text { ANT: Attention Network Test; HRT-SE: Hit Reaction Time Standard Error; } \mathrm{NO}_{2} \text { : Nitrogen Dioxide; } \mathrm{PM}_{2.5} \text { : Particulate matter } \\
<2.5 \mu \mathrm{m} ; \mathrm{BC}: \text { Black Carbon }\end{array}$} \\
\hline
\end{tabular}


Table 5. Stratified analysis of TRAPs concentration during commuting (only walkers) from home to school (interquartile range increase) on the 12-month change of outcomes of cognitive development ( working memory and inattentiveness). Adjusted models ${ }^{q}$

\begin{tabular}{|c|c|c|c|c|c|c|c|c|}
\hline & \multicolumn{2}{|c|}{ By School Air Pollution } & \multicolumn{2}{|c|}{ By Maternal Education } & \multicolumn{2}{|c|}{ By Sex } & \multicolumn{2}{|c|}{ By $A H^{\S}$} \\
\hline & $\begin{array}{l}\text { Low } \\
(n=369)\end{array}$ & $\begin{array}{c}\text { High } \\
(n=834)\end{array}$ & $\begin{array}{l}\text { Low } \\
(n=566)\end{array}$ & $\begin{array}{c}\text { High } \\
(n=637)\end{array}$ & $\begin{array}{l}\text { Male } \\
(n=602)\end{array}$ & $\begin{array}{l}\text { Female } \\
(n=601)\end{array}$ & $\begin{array}{c}\text { No } \\
(n=1,099)\end{array}$ & $\begin{array}{c}\text { Yes } \\
(n=120)\end{array}$ \\
\hline \multicolumn{9}{|c|}{ Working Memory (3-back Numbers, d') } \\
\hline $\mathrm{PM}_{2.5}$ & $\begin{array}{c}-4.4 \\
{[-12.8,4.0]}\end{array}$ & $\begin{array}{c}-2.0 \\
{[-9.6,5.6]}\end{array}$ & $\begin{array}{c}-6.2 \\
{[-12.6,0.2]}\end{array}$ & $\begin{array}{c}-5.1 \\
{[-12.3,2.0]}\end{array}$ & $\begin{array}{c}-9.2^{*} \\
{[-16.6,-1.8]}\end{array}$ & $\begin{array}{c}-2.1 \\
{[-8.3,4.0]}\end{array}$ & $\begin{array}{c}-6.0 * \\
{[-11.0,-1.0]}\end{array}$ & $\begin{array}{c}-4.9 \\
{[-20.2,10.4]}\end{array}$ \\
\hline BC & $\begin{array}{c}-1.4 \\
{[-10.4,7.5]}\end{array}$ & $\begin{array}{c}-2.2 \\
{[-9.1,4.8]}\end{array}$ & $\begin{array}{c}-4.5 \\
{[-10.6,1.5]}\end{array}$ & $\begin{array}{c}-4.8 \\
{[-11.5,1.8]}\end{array}$ & $\begin{array}{c}-9.7 * * \\
{[-16.8,-2.7]}\end{array}$ & $\begin{array}{c}-0.6 \\
{[-6.3,5.1]}\end{array}$ & $\begin{array}{c}-5.0 * \\
{[-9.8,-0.4]}\end{array}$ & $\begin{array}{c}-4.0 \\
{[-18.6,10.7]}\end{array}$ \\
\hline $\mathrm{NO}_{2}$ & $\begin{array}{c}-0.3 \\
{[-11.6,12.2]}\end{array}$ & $\begin{array}{c}0.8 \\
{[-6.0,7.7]}\end{array}$ & $\begin{array}{c}-3.1 \\
{[-10.0,3.7]}\end{array}$ & $\begin{array}{c}-2.7 \\
{[-10.3,4.8]}\end{array}$ & $\begin{array}{c}-6.5 \\
{[-14.2,1.3]}\end{array}$ & $\begin{array}{c}0.0 \\
{[-6.6,6.6]}\end{array}$ & $\begin{array}{c}-2.8 \\
{[-8.2,2.5]}\end{array}$ & $\begin{array}{c}-2.6 \\
{[-19.1,13.9]}\end{array}$ \\
\hline \multicolumn{9}{|c|}{ Inattentiveness (ANT, HRT-SE, milliseconds) } \\
\hline $\mathrm{PM}_{2.5}$ & $\begin{array}{c}-1.9 \\
{[-9.7,5.8]}\end{array}$ & $\begin{array}{c}2.4 \\
{[-4.3,9.0]}\end{array}$ & $\begin{array}{c}1.1 \\
{[-4.9,7.2]}\end{array}$ & $\begin{array}{c}4.2 \\
{[-2.0,10.3]}\end{array}$ & $\begin{array}{c}-0.2 \\
{[-6.7,6.2]}\end{array}$ & $\begin{array}{c}4.9 \\
{[-0.7,10.6]}\end{array}$ & $\begin{array}{c}2.1 \\
{[-2.4,6.6]}\end{array}$ & $\begin{array}{c}5.3 \\
{[-8.5,19.1]}\end{array}$ \\
\hline BC & $\begin{array}{c}-1.1 \\
{[-9.2,7.0]}\end{array}$ & $\begin{array}{c}1.3 \\
{[-4.8,7.4]}\end{array}$ & $\begin{array}{c}1.2 \\
{[-4.5,6.8]}\end{array}$ & $\begin{array}{c}3.5 \\
{[-2.1,9.1]}\end{array}$ & $\begin{array}{c}2.8 \\
{[-3.4,8.9]}\end{array}$ & $\begin{array}{c}2.1 \\
{[-3.1,7.3]}\end{array}$ & $\begin{array}{c}2.2 \\
{[-2.0,6.4]}\end{array}$ & $\begin{array}{c}3.7 \\
{[-9.5,16.9]}\end{array}$ \\
\hline $\mathrm{NO}_{2}$ & $\begin{array}{c}1.0 \\
{[-9.7,11.7]}\end{array}$ & $\begin{array}{c}-0.4 \\
{[-6.4,5.7]}\end{array}$ & $\begin{array}{c}2.6 \\
{[-3.8,9.0]}\end{array}$ & $\begin{array}{c}1.3 \\
{[-5.1,7.8]}\end{array}$ & $\begin{array}{c}3.2 \\
{[-3.6,10.0]}\end{array}$ & $\begin{array}{c}1.6 \\
{[-4.4,7.6]}\end{array}$ & $\begin{array}{c}1.8 \\
{[-2.9,6.6]}\end{array}$ & $\begin{array}{c}4.7 \\
{[-10.1,19.6]}\end{array}$ \\
\hline
\end{tabular}


573

574 
Table S2. Association between commuting concentration to tertiles of TRAPs (interquartile range increase) during commutting from home to school and neurodevelopmental outcomes. Adjusted models ${ }^{\ddagger}$.

\begin{tabular}{|c|c|c|}
\hline & $\begin{array}{c}\text { Working Memory } \\
\text { (3-back Numbers, } d^{\prime} \text { ) }\end{array}$ & $\begin{array}{c}\text { Inattentiveness } \\
\text { (ANT, HRT-SE, } \\
\text { milliseconds) }\end{array}$ \\
\hline \multicolumn{3}{|l|}{$\mathrm{PM}_{2.5}$} \\
\hline \multirow{2}{*}{$2^{\text {nd }}$ tertile } & -5.3 & -2.9 \\
\hline & {$[-13.7,3.0]$} & {$[-9.3,3.6]$} \\
\hline \multirow{2}{*}{$3^{\text {rd }}$ tertile } & $-20.1 * * *$ & 5.6 \\
\hline & {$[-29.5,-10.8]$} & {$[-2.0,13.3]$} \\
\hline \multicolumn{3}{|l|}{ BC } \\
\hline \multirow{2}{*}{$2^{\text {nd }}$ tertile } & -4.1 & -2.1 \\
\hline & {$[-12.6,4.4]$} & {$[-8.7,4.6]$} \\
\hline \multirow{2}{*}{$3^{\text {rd }}$ tertile } & $-19.3 * * *$ & 3.8 \\
\hline & {$[-28.8,-9.8]$} & {$[-4.0,11.5]$} \\
\hline \multicolumn{3}{|l|}{$\mathrm{NO}_{2}$} \\
\hline \multirow{2}{*}{$2^{\text {nd }}$ tertile } & -4.3 & -4.0 \\
\hline & {$[-12.8,4.1]$} & {$[-10.5,2.4]$} \\
\hline \multirow{2}{*}{$3^{\text {rd }}$ tertile } & -6.2 & -0.8 \\
\hline & {$[-15.7,3.3]$} & {$[-8.6,6.9]$} \\
\hline
\end{tabular}

$* \mathrm{p}<0.05 * * \mathrm{p}<0.01 * * * \mathrm{p}<0.001$

$\ddagger$ Difference in the 12-month change adjusted for age, sex, maternal education, residential neighborhood socio-economic status and air pollution during commuting; school and subject as nested random effects. ANT: Attention Network Test; HRT-SE: Hit Reaction Time Standard Error; $\mathrm{NO}_{2}$ : Nitrogen Dioxide; $\mathrm{PM}_{2.5}$ : Particulate matter <2.5 $\mu \mathrm{m}$; BC: Black Carbon Carbon

Linear trend $\mathrm{P}$-value $<0.05$ for $\mathrm{BC}$ and $\mathrm{PM}_{2.5}$ on superior working memory. 
Table S3. Difference (and $95 \%$ confidence interval) ${ }^{\ddagger}$ in the 12 -month change, per commuting air pollution exposure (concentration*time) during commuting (interquartile range increase, IQR) in 1.234 children. Air pollution concentrations estimated by LUR.

\begin{tabular}{|c|c|c|c|c|}
\hline \multicolumn{5}{|l|}{$\mathrm{PM}_{2.5}$ commuting } \\
\hline Exposure & $-6.8 * *$ & {$[-11.8,-1.7]$} & 0.4 & {$[-4.1,4.9]$} \\
\hline Exposure, adjusted for $\mathrm{PM}_{2.5}$ at school & $-7.0 * *$ & {$[-12.1,-1.9]$} & 0.3 & {$[-4.3,4.8]$} \\
\hline Exposure, adjusted for $\mathrm{PM}_{2.5}$ at home & $-6.8^{*}$ & {$[-11.9,-1.6]$} & 0.2 & {$[-4.4,4.8]$} \\
\hline Exposure, adjusted for $\mathrm{PM}_{2.5}$ at school and home & $-6.7^{*}$ & {$[-11.9,-1.5]$} & 0.2 & {$[-4.4,4.8]$} \\
\hline \multicolumn{5}{|l|}{ BC commutting } \\
\hline Exposure & $-7.0 * *$ & {$[-12.2,-1.8]$} & 1.3 & {$[-3.3,5.9]$} \\
\hline Exposure, adjusted for $\mathrm{PM}_{2.5}$ at school & $-7.6 * *$ & {$[-12.9,-2.2]$} & 1.1 & {$[-3.6,5.9]$} \\
\hline Exposure, adjusted for $\mathrm{PM}_{2.5}$ at home & $-7.5 * *$ & {$[-12.8,-2.1]$} & 1.3 & {$[-3.3,6.1]$} \\
\hline Exposure, adjusted for $\mathrm{PM}_{2.5}$ at school and home & $-7.6^{* *}$ & {$[13.0,-2.2]$} & 1.1 & {$[-3.6,5.9]$} \\
\hline \multicolumn{5}{|l|}{$\mathrm{NO}_{2}$ commuting } \\
\hline Exposure & $-5.2^{*}$ & {$[-10.0,-0.3]$} & 1.5 & {$[-2.9,5.8]$} \\
\hline Exposure, adjusted for $\mathrm{PM}_{2.5}$ at school & $-6.0^{*}$ & {$[-11.3,-0.8]$} & 1.3 & {$[-3.3,5.9]$} \\
\hline Exposure, adjusted for $\mathrm{PM}_{2.5}$ at home & -6.0 & {$[-11.3,-0.8]$} & 1.3 & {$[-3.3,5.9]$} \\
\hline \multicolumn{5}{|l|}{ Exposure, adjusted for $\mathrm{PM}_{2.5}$ at school and home } \\
\hline \multicolumn{5}{|c|}{$\begin{array}{l}{ }^{\ddagger} \text { Difference in the } 12-\text { month change adjusted for age, sex, maternal education and residential neighborhood socio- } \\
\text { economic status and other exposure when specified; school and subject as nested random effects. } \\
{ }^{\S} \text { Exposure }=\text { average concentration during commuting trip * trip time } \\
{ }^{*} \text { p }<0.05 * * p<0.01\end{array}$} \\
\hline \multicolumn{5}{|c|}{$\begin{array}{l}\text { ANT: Attention Network Test; HRT-SE: Hit Reaction Time Standard Error; } \mathrm{NO}_{2} \text { : Nitrogen Dioxide; } \mathrm{PM}_{2.5} \text { : Particulate } \\
\text { matter <2.5 } \mu \mathrm{m} \text {; BC: Black Carbon Carbon }\end{array}$} \\
\hline
\end{tabular}

